TRANSACTIONS OF THE

AMERICAN MATHEMATICAL SOCIETY

Volume 360, Number 6, June 2008, Pages 3211-3248

S 0002-9947(08)04465-6

Article electronically published on January 30, 2008

\title{
RIGID COMPLEXES VIA DG ALGEBRAS
}

\author{
AMNON YEKUTIELI AND JAMES J. ZHANG
}

\begin{abstract}
Let $A$ be a commutative ring, $B$ a commutative $A$-algebra and $M$ a complex of $B$-modules. We begin by constructing the square $\mathrm{Sq}_{B / A} M$, which is also a complex of $B$-modules. The squaring operation is a quadratic functor, and its construction requires differential graded (DG) algebras. If there exists an isomorphism $\rho: M \stackrel{\simeq}{\longrightarrow} \operatorname{Sq}_{B / A} M$, then the pair $(M, \rho)$ is called a rigid complex over $B$ relative to $A$ (there are some finiteness conditions). There is an obvious notion of rigid morphism between rigid complexes.

We establish several properties of rigid complexes, including their uniqueness, existence (under some extra hypothesis), and formation of pullbacks $f^{b}(M, \rho)$ (resp. $f^{\sharp}(M, \rho)$ ) along a finite (resp. essentially smooth) ring homomorphism $f^{*}: B \rightarrow C$.

In the subsequent paper, Rigid Dualizing Complexes over Commutative Rings, we consider rigid dualizing complexes over commutative rings, building on the results of the present paper. The project culminates in our paper Rigid Dualizing Complexes and Perverse Sheaves on Schemes, where we give a comprehensive version of Grothendieck duality for schemes.

The idea of rigid complexes originates in noncommutative algebraic geometry, and is due to Van den Bergh (1997).
\end{abstract}

\section{INTRODUCTION}

Rigid dualizing complexes were invented by Van den Bergh [VdB] in the context of noncommutative algebraic geometry. Since then the theory of rigid dualizing complexes was developed further by several people, with many applications in the areas of noncommutative algebra and noncommutative algebraic geometry. See the papers [YZ1, YZ2, YZ3] and their references.

The present paper is the first in a series of three papers in which we apply the rigidity technique to the areas of commutative algebra and algebraic geometry. Of course the commutative setup is "contained in", and is always much "easier" than the corresponding noncommutative setup. The point is that we want to get stronger and more general results when we restrict our attention to commutative rings and schemes.

Let us remind the reader that in the papers cited above the noncommutative algebras in question were always assumed to be over some base field $\mathbb{K}$. The first generalization that comes to mind when considering a commutative theory of rigid dualizing complexes is to be able to work over any commutative base ring $\mathbb{K}$, or

Received by the editors June 22, 2006.

2000 Mathematics Subject Classification. Primary 18E30; Secondary 18G10, 16E45, 18 G15.

Key words and phrases. Commutative rings, DG algebras, derived categories, rigid complexes.

This research was supported by the US-Israel Binational Science Foundation. The second author was partially supported by the US National Science Foundation. 
at least a sufficiently nice base ring, such as $\mathbb{K}=\mathbb{Z}$ for instance. This seemingly innocent generalization turns out to be quite hard. The present paper is devoted to solving it, by developing the technique of rigid complexes over commutative rings. The subsequent papers [YZ4, Ye4] will build on the foundations laid here, and will produce a comprehensive theory of dualizing complexes (including duality for proper morphisms) parallel to that of [RD], for finite type schemes over a regular finite dimensional commutative base ring $\mathbb{K}$.

It should be noted that the rigidity theory developed here is essential even when dealing with commutative algebras (or schemes) over a base field $\mathbb{K}$. This is because it enables the concept of relative rigid complex, with respect to a $\mathbb{K}$-algebra homomorphism $A \rightarrow B$.

For the rest of the Introduction let us make the blanket assumption that all rings occurring are commutative. As usual, by an algebra over a $\operatorname{ring} A$ we mean a ring $B$ together with a ring homomorphism $A \rightarrow B$. We denote by $\mathrm{D}(\operatorname{Mod} B)$ the derived category of $B$-modules. If $B$ is noetherian, then we also consider the full subcategory $\mathrm{D}_{\mathrm{f}}^{\mathrm{b}}(\operatorname{Mod} B)$ consisting of bounded complexes with finitely generated cohomologies.

The difficulty in generalizing rigidity to an arbitrary base ring $A$ already shows up in the definition of rigidity (Van den Bergh's rigidity equation). Let us first recall the original definition from $[\mathrm{VdB}]$. Suppose $A$ is a field, $B$ is a noetherian $A$-algebra and $M \in \mathrm{D}_{\mathrm{f}}^{\mathrm{b}}(\operatorname{Mod} B)$. A rigidifying isomorphism for $M$ is an isomorphism

$$
\rho: M \stackrel{\simeq}{\longrightarrow} \operatorname{RHom}_{B \otimes_{A} B}\left(B, M \otimes_{A} M\right)
$$

in $\mathrm{D}(\operatorname{Mod} B)$. The pair $(M, \rho)$ is called a rigid complex over $B$ relative to $A$.

Now suppose $A$ is any ring, and $B$ is a noetherian $A$-algebra which is not flat. Then in (0.1) we must replace $M \otimes_{A} M$ with $M \otimes_{A}^{\mathrm{L}} M$; but $M \otimes_{A}^{\mathrm{L}} M$ is not usually a well-defined object in the derived category $\mathrm{D}\left(\operatorname{Mod} B \otimes_{A} B\right) \ldots$

Our solution is to use differential graded (DG) algebras. Take any ring $A$ and any $A$-algebra $B$. Choose some semi-free super-commutative nonpositive DG algebra resolution $\tilde{B} \rightarrow B$ relative to $A$. (All necessary facts about DG algebras are either reviewed or proved in Section 1 of the paper.) Let $M \in \mathrm{D}(\operatorname{Mod} B)$. We prove that the complex

$$
\mathrm{Sq}_{B / A} M:=\operatorname{RHom}_{\tilde{B} \otimes_{A} \tilde{B}}\left(B, M \otimes_{A}^{\mathrm{L}} M\right) \in \mathrm{D}(\operatorname{Mod} B)
$$

is independent of the resolution $\tilde{B} \rightarrow B$. Moreover,

$$
\mathrm{Sq}_{B / A}: \mathrm{D}(\operatorname{Mod} B) \rightarrow \mathrm{D}(\operatorname{Mod} B)
$$

is a (nonlinear) functor. See Theorems 2.2 and 2.3 .

We are now ready to define rigidity. Let $A$ be a ring, let $B$ be a noetherian $A$-algebra and let $M \in \mathrm{D}_{\mathrm{f}}^{\mathrm{b}}(\operatorname{Mod} B)$. Assume $M$ has finite flat dimension over $A$. (This latter condition is automatically satisfied if $A$ is a regular ring.) Then, just like (0.1), a rigidifying isomorphism for $M$ is an isomorphism

$$
\rho: M \stackrel{\simeq}{\longrightarrow} \mathrm{Sq}_{B / A} M
$$

in $\mathrm{D}(\operatorname{Mod} B)$, and the pair $(M, \rho)$ is called a rigid complex over $B$ relative to $A$. 
Suppose we are given two rigid complexes $\left(M, \rho_{M}\right)$ and $\left(N, \rho_{N}\right)$ over $B$ relative to $A$. A morphism $\phi: M \rightarrow N$ in $\mathrm{D}(\operatorname{Mod} B)$ is said to be rigid relative to $A$ if the diagram

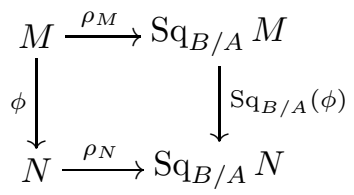

is commutative. The category of such rigid complexes and rigid morphisms is denoted by $\mathrm{D}_{\mathrm{f}}^{\mathrm{b}}(\operatorname{Mod} B)_{\mathrm{rig} / A}$.

The following result about rigid complexes explains their name.

Theorem 0.2. Let $A$ be a ring, let $B$ be a noetherian $A$-algebra, and let $(M, \rho) \in$ $\mathrm{D}_{\mathrm{f}}^{\mathrm{b}}(\operatorname{Mod} B)_{\mathrm{rig} / A}$. Assume the canonical homomorphism $B \rightarrow \operatorname{Hom}_{\mathrm{D}(\operatorname{Mod} B)}(M, M)$ is bijective. Then the only automorphism of $(M, \rho)$ in $\mathrm{D}_{\mathrm{f}}^{\mathrm{b}}(\operatorname{Mod} B)_{\mathrm{rig} / A}$ is the identity $\mathbf{1}_{M}$.

This result is repeated as Theorem 4.4 in the body of the paper. The proof boils down to the the following fact: let $\phi: M \rightarrow M$ be an isomorphism in $\mathrm{D}(\operatorname{Mod} B)$, so $\phi=b \mathbf{1}_{M}$ for some invertible element $b \in B$. If $\phi$ is rigid, then a calculation shows that $b^{2}=b$; and hence $b=1$.

We find it convenient to denote ring homomorphisms by $f^{*}$, etc. Thus a ring homomorphism $f^{*}: A \rightarrow B$ corresponds to a morphism of schemes $f: \operatorname{Spec} B \rightarrow$ $\operatorname{Spec} A$.

Let $A$ be a noetherian ring. Recall that an $A$-algebra $B$ is called an essentially finite type if it is a localization of some finitely generated $A$-algebra. We say that $B$ is essentially smooth (resp. essentially étale) over $A$ if it is of essentially finite type and formally smooth (resp. formally étale). These concepts are studied in Section 3.

Let $A$ be a noetherian ring and $f^{*}: A \rightarrow B$ an essentially smooth homomorphism. Then $\Omega_{B / A}^{1}$ is a finitely generated projective $B$-module. Let $\operatorname{Spec} B=$ $\coprod_{i}$ Spec $B_{i}$ be the decomposition into connected components, and for every $i$ let $n_{i}$ be the rank of $\Omega_{B_{i} / A}^{1}$. We define a functor

$$
f^{\sharp}: \mathrm{D}(\operatorname{Mod} A) \rightarrow \mathrm{D}(\operatorname{Mod} B)
$$

by

$$
f^{\sharp} M:=\bigoplus_{i} \Omega_{B_{i} / A}^{n_{i}}\left[n_{i}\right] \otimes_{A} M .
$$

Recall that a ring homomorphism $f^{*}: A \rightarrow B$ is called finite if $B$ is a finitely generated $A$-module. Given such a finite homomorphism we define a functor

$$
f^{b}: \mathrm{D}(\operatorname{Mod} A) \rightarrow \mathrm{D}(\operatorname{Mod} B)
$$

by

$$
f^{b} M:=\operatorname{RHom}_{A}(B, M) .
$$

Theorem 0.3. Let $A$ be a noetherian ring, let $B, C$ be essentially finite type $A$-algebras, let $f^{*}: B \rightarrow C$ be an A-algebra homomorphism, and let $(M, \rho) \in$ $\mathrm{D}_{\mathrm{f}}^{\mathrm{b}}(\operatorname{Mod} B)_{\mathrm{rig} / A}$. 
(1) If $f^{*}$ is finite and $f^{b} M$ has finite flat dimension over $A$, then $f^{b} M$ has an induced rigidifying isomorphism

$$
f^{b}(\rho): f^{b} M \stackrel{\simeq}{\longrightarrow} \mathrm{Sq}_{C / A} f^{b} M .
$$

The rigid complex $f^{b}(\rho)(M, \rho):=\left(f^{b} M, f^{b}(\rho)\right)$ depends functorially on $(M, \rho)$ and on $f^{*}$.

(2) If $f^{*}$ is essentially smooth, then $f^{\sharp} M$ has an induced rigidifying isomorphism

$$
f^{\sharp}(\rho): f^{\sharp} M \stackrel{\simeq}{\longrightarrow} \mathrm{Sq}_{C / A} f^{\sharp} M .
$$

The rigid complex $f^{\sharp}(\rho)(M, \rho):=\left(f^{\sharp} M, f^{\sharp}(\rho)\right)$ depends functorially on $(M, \rho)$ and on $f^{*}$.

This result is included in Theorems 5.3 and 6.3 in the body of the paper.

The next result is about tensor products of rigid complexes.

Theorem 0.4. Let $A$ be a noetherian ring, let $B, C$ be essentially finite type $A$ algebras, let $f^{*}: B \rightarrow C$ be an A-algebra homomorphism, and let $\left(M, \rho_{M}\right) \in$ $\mathrm{D}_{\mathrm{f}}^{\mathrm{b}}(\operatorname{Mod} B)_{\mathrm{rig} / A}$ and $\left(N, \rho_{N}\right) \in \mathrm{D}_{\mathrm{f}}^{\mathrm{b}}(\operatorname{Mod} C)_{\mathrm{rig} / B}$. Assume that the canonical homomorphism $B \rightarrow \operatorname{Hom}_{\mathrm{D}(\operatorname{Mod} B)}(M, M)$ is bijective. Then the complex $M \otimes_{B}^{\mathrm{L}} N$ is in $\mathrm{D}_{\mathrm{f}}^{\mathrm{b}}(\operatorname{Mod} C)$, it has finite flat dimension over $A$, and it has an induced rigidifying isomorphism

$$
\rho_{M} \otimes \rho_{N}: M \otimes_{B}^{\mathrm{L}} N \stackrel{\simeq}{\longrightarrow} \operatorname{Sq}_{C / A}\left(M \otimes_{B}^{\mathrm{L}} N\right) .
$$

This is part of Corollary 4.13.

So far we said nothing about existence of rigid complexes. We can do so under some extra hypothesis.

Theorem 0.5. Let $\mathbb{K}$ be a regular noetherian ring of finite Krull dimension, and let $A$ be an essentially finite type $\mathbb{K}$-algebra. Then $A$ has a rigid complex $(R, \rho)$ relative to $\mathbb{K}$, such that the canonical homomorphism $A \rightarrow \operatorname{Hom}_{\mathrm{D}(\operatorname{Mod} A)}(R, R)$ is bijective.

This theorem is repeated as Theorem 6.16 in the body of the paper. Note that $R$ is nonzero (if $A \neq 0$ ), and moreover, by Theorem 0.2 , the only rigid automorphism of $(R, \rho)$ is the identity. The construction of the rigid complex $(R, \rho)$ is actually quite easy (given Theorem 0.3).

The next theorem, which combines several results from our paper [YZ4], gives a precise description of all rigid complexes in this situation. Recall that a dualizing complex $[\mathrm{RD}]$ is a complex $R \in \mathrm{D}_{\mathrm{f}}^{\mathrm{b}}(\operatorname{Mod} A)$ which has finite injective dimension, and the canonical morphism $A \rightarrow \operatorname{RHom}_{A}(R, R)$ is an isomorphism.

Theorem 0.6 ([YZ4]). Suppose $\mathbb{K}$ is a regular noetherian ring of finite Krull dimension. Let $A$ be an essentially finite type $\mathbb{K}$-algebra, such that $\mathrm{Spec} A$ is connected and nonempty. Then, up to isomorphism, the category $\mathrm{D}_{\mathrm{f}}^{\mathrm{b}}(\operatorname{Mod} A)_{\mathrm{rig} / \mathbb{K}}$ has exactly two objects: the zero complex, and the nonzero rigid complex $(R, \rho)$ from Theorem 0.5. Moreover, $R$ is a dualizing complex over $A$.

We find Theorem 0.6 very surprising, and we do not understand its significance yet. Also we do not know whether a similar result holds in the noncommutative setup.

To end the Introduction let us mention a couple of other papers that approach Grothendieck duality in novel ways: [Ne] and [DGI]. 


\section{Differential graded algebras}

This section contains some technical material about differential graded algebras and their derived module categories. There is some overlap here with the papers [FIJ], [Ke] and [Be].

A graded algebra $A=\bigoplus_{i \in \mathbb{Z}} A^{i}$ is said to be super-commutative if $a \cdot b=(-1)^{i j} b \cdot a$ for all $a \in A^{i}$ and $b \in A^{j}$, and if $a \cdot a=0$ whenever $i$ is odd. (Some authors call such a graded algebra strictly commutative.) The graded algebra $A$ is said to be nonpositive if $A^{i}=0$ for all $i>0$.

Convention 1.1. Throughout the paper all graded algebras are assumed by default to be nonpositive, super-commutative, associative and unital. Homomorphisms of graded algebras are unital and have degree 0.

Suppose $A \rightarrow B$ is a homomorphism of graded algebras. Then we say that $B$ is a graded $A$-algebra.

By differential graded algebra (or DG algebra) we mean a graded algebra $A=$ $\bigoplus_{i \leq 0} A^{i}$, together with a derivation $\mathrm{d}: A \rightarrow A$ of degree 1 satisfying $\mathrm{d} \circ \mathrm{d}=0$. Note that the graded Leibniz rule holds:

$$
\mathrm{d}(a b)=\mathrm{d}(a) b+(-1)^{i} a \mathrm{~d}(b)
$$

for $a \in A^{i}$ and $b \in A^{j}$. The cohomology $\mathrm{H} A=\bigoplus_{i<0} \mathrm{H}^{i} A$ is then a graded algebra.

All rings in this paper are considered as DG algebras concentrated in degree 0 (with zero differential); in particular rings are assumed to be commutative.

A DG algebra homomorphism $u: A \rightarrow B$ is a homomorphism of graded algebras that commutes with $\mathrm{d}$. It is a quasi-isomorphism if $\mathrm{H}(u): \mathrm{H} A \rightarrow \mathrm{H} B$ is an isomorphism (of graded algebras).

If $A$ is a ring, $B$ is a DG algebra, and we are given a DG algebra homomorphism $A \rightarrow B$, then the differential $\mathrm{d}_{B}$ is necessarily $A$-linear. In this case we say that $B$ is a DG $A$-algebra. If $B=B^{0}$, i.e. $B$ is a ring too, then we say that $B$ is an $A$-algebra.

A differential graded (DG) $A$-module is a graded (left) $A$-module $M=\bigoplus_{i \in \mathbb{Z}} M^{i}$, endowed with a degree $1 \mathbb{Z}$-linear homomorphism d $: M \rightarrow M$ satisfying $\mathrm{d}(\mathrm{am})=$ $\mathrm{d}(a) m+(-1)^{i} a \mathrm{~d}(m)$ for $a \in A^{i}$ and $m \in M^{j}$. Note that we can make $M$ into a right DG $A$-module by the rule $m a:=(-1)^{i j} a m$ for $a \in A^{i}$ and $m \in M^{j}$. The category of DG $A$-modules is denoted by DGMod $A$. It is an abelian category whose morphisms are degree $0 A$-linear homomorphisms commuting with the differentials.

There is a forgetful functor from DG algebras to graded algebras (it forgets the differential), and we denote it by $A \mapsto$ und $A$. Likewise for $M \in \operatorname{DGMod} A$ we have und $M \in \operatorname{GrMod}($ und $A$ ), the category of graded und $A$-modules. If $A$ is a ring, then a DG $A$-module is just a complex of $A$-modules.

Given a graded algebra $A$ and two graded $A$-modules $M$ and $N$ let us write

$$
\operatorname{Hom}_{\mathbb{Z}}(M, N)^{i}:=\prod_{j \in \mathbb{Z}} \operatorname{Hom}_{\mathbb{Z}}\left(M^{j}, N^{j+i}\right),
$$

the set of homogeneous $\mathbb{Z}$-linear homomorphisms of degree $i$ from $M$ to $N$, and let

$$
\begin{aligned}
& \operatorname{Hom}_{A}(M, N)^{i} \\
& \quad:=\left\{\phi \in \operatorname{Hom}_{\mathbb{Z}}(M, N)^{i} \mid \phi(a m)=(-1)^{i j} a \phi(m) \text { for all } a \in A^{j} \text { and } m \in M\right\} .
\end{aligned}
$$


Then

$$
\operatorname{Hom}_{A}(M, N):=\bigoplus_{i \in \mathbb{Z}} \operatorname{Hom}_{A}(M, N)^{i}
$$

is a graded $A$-module, by the formula $(a \phi)(m):=a \phi(m)=(-1)^{i j} \phi(a m)$ for $a \in A^{j}$ and $\phi \in \operatorname{Hom}_{A}(M, N)^{i}$. Cf. [ML, Chapter VI]. The set $\operatorname{Hom}_{A}(M, N)$ is related to the set of $A$-linear homomorphisms $M \rightarrow N$ as follows. Let's denote by ungr the functor forgetting the grading. Then the map

$$
\Phi: \operatorname{Hom}_{A}(M, N) \rightarrow \operatorname{Hom}_{\text {ungr } A}(\operatorname{ungr} M, \operatorname{ungr} N),
$$

defined by $\Phi(\phi)(m):=(-1)^{i j} \phi(m)$ for $\phi \in \operatorname{Hom}_{A}(M, N)^{i}$ and $m \in M^{j}$, is ungr $A$ linear, and $\Phi$ is bijective if $M$ is a finitely generated $A$-module.

For a DG algebra $A$ and two DG $A$-modules $M, N$ there is a differential d on $\operatorname{Hom}_{\text {und } A}($ und $M$, und $N)$, with formula $\mathrm{d}(\phi):=\mathrm{d} \circ \phi-(-1)^{i} \phi \circ \mathrm{d}$ for $\phi$ of degree $i$. The resulting DG $A$-module is denoted by $\operatorname{Hom}_{A}(M, N)$. Note that $\operatorname{Hom}_{\mathrm{DGMod} A}(M, N)$ coincides with the set of 0-cocycles of $\operatorname{Hom}_{A}(M, N)$. Two homomorphisms $\phi_{0}, \phi_{1} \in \operatorname{Hom}_{\mathrm{DGMod} A}(M, N)$ are said to be homotopic if $\phi_{0}-\phi_{1}=$ $\mathrm{d}(\psi)$ for some $\psi \in \operatorname{Hom}_{A}(M, N)^{-1}$. The DG modules $M$ and $N$ are called homotopy equivalent if there are homomorphisms $\phi: M \rightarrow N$ and $\psi: N \rightarrow M$ in DGMod $A$ such that $\psi \circ \phi$ and $\phi \circ \psi$ are homotopic to the respective identity homomorphisms.

Suppose $A$ is a ring and $B, C$ are two DG $A$-algebras. Then $B \otimes_{A} C$ is also a DG $A$-algebra; the sign rule says that

$$
\left(b_{1} \otimes c_{1}\right) \cdot\left(b_{2} \otimes c_{2}\right):=(-1)^{i j} b_{1} b_{2} \otimes c_{1} c_{2}
$$

for $c_{1} \in C^{j}$ and $b_{2} \in B^{i}$. The differential is of course

$$
\mathrm{d}(b \otimes c):=\mathrm{d}(b) \otimes c+(-1)^{i} b \otimes \mathrm{d}(c)
$$

for $b \in B^{i}$. If $M \in \mathrm{DGMod} B$ and $N \in \operatorname{DGMod} C$, then $M \otimes_{A} N \in \operatorname{DGMod} B \otimes_{A} C$. If $N \in \operatorname{DGMod} B$, then $M \otimes_{B} N$, which is a quotient of $M \otimes_{A} N$, is a DG $B$-module.

Let $A$ be a DG algebra. Since $A$ is nonpositive one has $\mathrm{d}\left(A^{0}\right)=0$, and therefore the differential d : $M^{i} \rightarrow M^{i+1}$ of any DG $A$-module $M$ is $A^{0}$-linear. This easily implies that the truncated objects

$$
\begin{aligned}
& \tau^{\geq i} M:=\left(\cdots 0 \rightarrow \operatorname{Coker}\left(M^{i-1} \rightarrow M^{i}\right) \rightarrow M^{i+1} \rightarrow \cdots\right) \\
& \quad \text { and } \\
& \tau^{\leq i} M:=\left(\cdots \rightarrow M^{i-1} \rightarrow \operatorname{Ker}\left(M^{i} \rightarrow M^{i+1}\right) \rightarrow 0 \rightarrow \cdots\right)
\end{aligned}
$$

are DG $A$-modules.

There is a derived category obtained from DGMod $A$ by inverting the quasi-isomorphisms, which we denote by $\tilde{\mathrm{D}}(\operatorname{DGMod} A)$. See $[\mathrm{Ke}]$ for details. Note that in case $A$ is a ring then $\mathrm{DGMod} A=\mathrm{C}(\operatorname{Mod} A)$, the abelian category of complexes of $A$ modules, and $\tilde{\mathrm{D}}(\operatorname{DGMod} A)=\mathrm{D}(\operatorname{Mod} A)$, the usual derived category of $A$-modules (as in $[\mathrm{RD}]$ or $[\mathrm{KS}]$ ).

In order to derive functors one has several useful devices. A DG $A$-module $P$ is called $K$-projective if for any acyclic DG $A$-module $N$ the DG module $\operatorname{Hom}_{A}(P, N)$ is acyclic. (This name is due to Spaltenstein [Sp]. Keller [Ke] uses the term "property (P)" to indicate K-projective DG modules, and in $[\mathrm{AFH}]$ the authors use "homotopically projective". See also [Hi].) Similarly one defines K-injective and $K$-flat DG modules: $I$ is K-injective, and $F$ is $\mathrm{K}$-flat, if $\operatorname{Hom}_{A}(N, I)$ and $F \otimes_{A} N$ are acyclic for all acyclic $N$. It is easy to see that any K-projective DG module is also K-flat. Every two objects $M, N \in \operatorname{DGMod} A$ admit quasi-isomorphisms $P \rightarrow M$, 
$N \rightarrow I$ and $F \rightarrow M$, with $P$ K-projective, $I$ K-injective and $F$ K-flat. Then one defines

$$
\operatorname{RHom}_{A}(M, N):=\operatorname{Hom}_{A}(P, N) \cong \operatorname{Hom}_{A}(M, I) \in \tilde{\mathrm{D}}(\mathrm{DGMod} A)
$$

and

$$
M \otimes_{A}^{\mathrm{L}} N:=F \otimes_{A} N \in \tilde{\mathrm{D}}(\operatorname{DGMod} A) .
$$

When $A$ is a usual algebra, any bounded above complex of projective (resp. flat) modules is K-projective (resp. K-flat), and any bounded below complex of injective $A$-modules is $\mathrm{K}$-injective. A single $A$-module $M$ is projective (resp. injective, resp. flat) iff it is K-projective (resp. K-injective, resp. K-flat) as a DG $A$-module.

The following useful result is partly contained in $[\mathrm{Hi}]$, [Ke] and $[\mathrm{KM}]$.

Proposition 1.4. Let $A \rightarrow B$ be a quasi-isomorphism of $D G$ algebras.

(1) Given $M \in \tilde{\mathrm{D}}(\mathrm{DGMod} A)$ and $N \in \tilde{\mathrm{D}}(\mathrm{DGMod} B)$, the canonical morphisms $M \rightarrow B \otimes{ }_{A}^{\mathrm{L}} M$ and $B \otimes{ }_{A}^{\mathrm{L}} N \rightarrow N$ are both isomorphisms. Hence the "restriction of scalars" functor $\tilde{\mathrm{D}}(\mathrm{DGMod} B) \rightarrow \tilde{\mathrm{D}}(\mathrm{DGMod} A)$ is an equivalence.

(2) Let $M, N \in \tilde{\mathrm{D}}(\mathrm{DGMod} B)$. Then there are functorial isomorphisms $M \otimes_{B}^{\mathrm{L}}$ $N \cong M \otimes_{A}^{\mathrm{L}} N$ and $\operatorname{RHom}_{B}(M, N) \cong \operatorname{RHom}_{A}(M, N)$ in $\tilde{\mathrm{D}}(\operatorname{DGMod} A)$.

Proof. (1) Choose K-projective resolutions $P \rightarrow M$ and $Q \rightarrow N$ over $A$. Then $M \rightarrow B \otimes_{A}^{\mathrm{L}} M$ becomes $P \cong A \otimes_{A} P \rightarrow B \otimes_{A} P$, which is evidently a quasiisomorphism. On the other hand $B \otimes_{A}^{\mathrm{L}} N \rightarrow N$ becomes $B \otimes_{A} Q \rightarrow Q$, which is a quasi-isomorphism because so is $A \otimes_{A} Q \rightarrow B \otimes_{A} Q$.

(2) Choose K-projective resolutions $P \rightarrow M$ and $Q \rightarrow N$ over $A$. We note that $B \otimes_{A} P$ and $B \otimes_{A} Q$ are K-projective over $B$, and $B \otimes_{A} P \rightarrow M, B \otimes_{A} Q \rightarrow N$ are quasi-isomorphisms. Therefore we get isomorphisms in $\tilde{\mathrm{D}}(\operatorname{DGMod} A)$ :

$$
M \otimes_{B}^{\mathrm{L}} N=\left(B \otimes_{A} P\right) \otimes_{B}\left(B \otimes_{A} Q\right) \cong\left(B \otimes_{A} P\right) \otimes_{A} Q \cong P \otimes_{A} Q=M \otimes_{A}^{\mathrm{L}} N .
$$

The same resolutions give

$$
\operatorname{RHom}_{B}(M, N)=\operatorname{Hom}_{B}\left(B \otimes_{A} P, N\right) \cong \operatorname{Hom}_{A}(P, N)=\operatorname{RHom}_{A}(M, N) .
$$

There is a structural characterization of K-projective DG modules, which we shall review (since we shall elaborate on it later). This characterization works in steps. First one defines semi-free DG $A$-modules. A DG $A$-module $Q$ is called semi-free if there is a subset $X \subset Q$ consisting of (nonzero) homogeneous elements, and an exhaustive nonnegative increasing filtration $\left\{F_{i} X\right\}_{i \in \mathbb{Z}}$ of $X$ by subsets (i.e. $F_{-1} X=\emptyset$ and $\left.X=\bigcup F_{i} X\right)$, such that und $Q$ is a free graded und $A$-module with basis $X$, and for every $i$ one has $\mathrm{d}\left(F_{i} X\right) \subset \sum_{x \in F_{i-1} X} A x$. The set $X$ is called a semi-basis of $Q$. Note that $X$ is partitioned into $X=\coprod_{i \in \mathbb{Z}} X_{i}$, where $X_{i}:=X \cap Q^{i}$. We call such a set a graded set. Now a DG $A$-module $P$ is K-projective iff it is homotopy equivalent to a direct summand (in DGMod $A$ ) of some semi-free DG module $Q$. See $[\mathrm{AFH}]$ or $[\mathrm{Ke}]$ for more details and for proofs.

A free (super-commutative, nonpositive) graded $\mathbb{Z}$-algebra is a graded algebra of the following form. One starts with a graded set of variables $X=\coprod_{i \leq 0} X_{i}$; the elements of $X_{i}$ are the variables of degree $i$. Let $X_{\mathrm{ev}}:=\coprod_{i \text { even }} X_{i}$ and $X_{\text {odd }}:=$ $\coprod_{i \text { odd }} X_{i}$. Consider the free associative $\mathbb{Z}$-algebra $\mathbb{Z}\langle X\rangle$ on this set of variables. Let $I$ be the two-sided ideal of $\mathbb{Z}\langle X\rangle$ generated by all elements of the form $x y-(-1)^{i j} y x$ 
or $z^{2}$, where $x \in X_{i}, y \in X_{j}, z \in X_{k}$, and $k$ is odd. The free super-commutative graded $\mathbb{Z}$-algebra on $X$ is the quotient $\mathbb{Z}[X]:=\mathbb{Z}\langle X\rangle / I$. It is useful to note that

$$
\mathbb{Z}[X] \cong \mathbb{Z}\left[X_{\text {ev }}\right] \otimes_{\mathbb{Z}} \mathbb{Z}\left[X_{\text {odd }}\right]
$$

and that $\mathbb{Z}\left[X_{\text {ev }}\right]$ is a commutative polynomial algebra, whereas $\mathbb{Z}\left[X_{\text {odd }}\right]$ is an exterior algebra.

Definition 1.5. Suppose $A \rightarrow B$ is a homomorphism of DG algebras. $B$ is called a semi-free (super-commutative, nonpositive) $D G$ algebra relative to $A$ if there is a graded set $X=\coprod_{i \leq 0} X_{i}$, and an isomorphism of graded und $A$-algebras

$$
\text { (und } A) \otimes_{\mathbb{Z}} \mathbb{Z}[X] \cong \text { und } B \text {. }
$$

Observe that the DG algebra $B$ in the definition above, when regarded as a DG $A$-module, is semi-free with semi-basis consisting of the monomials in elements of $X$. Hence $B$ is also $\mathrm{K}$-projective and $\mathrm{K}$-flat as a DG $A$-module.

Definition 1.6. Suppose $A$ and $B$ are DG algebras and $u: A \rightarrow B$ is a homomorphism of DG algebras. A semi-free (resp. K-projective, resp. K-flat) DG algebra resolution of $B$ relative to $A$ is the data $A \stackrel{\tilde{u}}{\rightarrow} \tilde{B} \stackrel{v}{\rightarrow} B$, where $\tilde{B}$ is a DG $\mathbb{K}$-algebra, $\tilde{u}$ and $v$ are $\mathrm{DG}$ algebra homomorphisms, and the following conditions are satisfied:

(i) $v \circ \tilde{u}=u$.

(ii) $v$ is a quasi-isomorphism.

(iii) $\tilde{u}$ makes $\tilde{B}$ into a semi-free DG algebra relative to $A$ (resp. a K-projective DG $A$-module, resp. a K-flat DG $A$-module).

We also say that $A \stackrel{\tilde{u}}{\rightarrow} \tilde{B} \stackrel{v}{\rightarrow} B$ is a semi-free (resp. K-projective, resp. K-flat) DG algebra resolution of $A \stackrel{u}{\rightarrow} B$ :

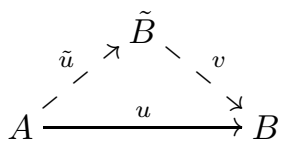

Proposition 1.7. Let $A$ and $B$ be $D G$ algebras, and let $u: A \rightarrow B$ be a DG algebra homomorphism.

(1) There exists a semi-free $D G$ algebra resolution $A \stackrel{\tilde{u}}{\rightarrow} \tilde{B} \stackrel{v}{\rightarrow} B$ of $A \stackrel{u}{\rightarrow} B$.

(2) Moreover, if $\mathrm{H} A$ is a noetherian algebra and $\mathrm{H} B$ is a finitely generated $\mathrm{H} A$ algebra, then we can choose the semi-free DG algebra $\tilde{B}$ in part (1) such that und $\tilde{B} \cong($ und $A) \otimes_{\mathbb{Z}} \mathbb{Z}[X]$ as graded und $A$-algebras, where the graded set $X=\coprod_{i \leq 0} X_{i}$ has finite graded components $X_{i}$.

(3) If $\mathrm{H} A$ is a noetherian algebra, $B$ is a ring, and $B=\mathrm{H}^{0} B$ is a finitely generated $\mathrm{H}^{0} A$-module, then there exists a K-projective $D G$ algebra resolution $A \rightarrow \tilde{B} \rightarrow B$ of $A \rightarrow B$, such that und $\tilde{B} \cong \bigoplus_{i=-\infty}^{0}$ und $A[-i]^{\mu_{i}}$ as graded und $A$-modules, and the multiplicities $\mu_{i}$ are finite.

Proof. (1) We shall construct $\tilde{B}$ as the union of an increasing sequence of DG algebras $F_{0} \tilde{B} \subset F_{1} \tilde{B} \subset \cdots$, which will be defined recursively. At the same time we shall construct an increasing sequence of DG algebra homomorphisms $A \rightarrow F_{i} \tilde{B} \stackrel{v_{i}}{\longrightarrow}$ $B$, and an increasing sequence of graded sets $F_{i} X \subset F_{i} \tilde{B}$. The homomorphism $v$ will be the union of the $v_{i}$, and the graded set $X=\coprod_{j \leq 0} X_{j}$ will be the union of the sets $F_{i} X$. For every $i$ the following conditions will hold. 
(i) $\mathrm{H}\left(v_{i}\right): \mathrm{H}\left(F_{i} \tilde{B}\right) \rightarrow \mathrm{H} B$ is surjective in degrees $\geq-i$.

(ii) $\mathrm{H}\left(v_{i}\right): \mathrm{H}\left(F_{i} \tilde{B}\right) \rightarrow \mathrm{H} B$ is bijective in degrees $\geq-i+1$.

(iii) $F_{i} \tilde{B}=A\left[F_{i} X\right], \mathrm{d}\left(F_{i} X\right) \subset F_{i-1} \tilde{B}$ and und $F_{i} \tilde{B} \cong($ und $A) \otimes_{\mathbb{Z}} \mathbb{Z}\left[F_{i} X\right]$.

We start by choosing a set of elements of $B^{0}$ that generate $\mathrm{H}^{0} B$ as an $\mathrm{H}^{0} A$ algebra. This gives us a set $Y_{0}$ of elements of degree 0 with a function $v_{0}: Y_{0} \rightarrow B^{0}$. Consider the DG algebra $\mathbb{Z}\left[Y_{0}\right]$ with zero differential, and define $F_{0} \tilde{B}:=A \otimes_{\mathbb{Z}} \mathbb{Z}\left[Y_{0}\right]$. Also define $F_{0} X:=Y_{0}$. We get a DG algebra homomorphism $v_{0}: F_{0} \tilde{B} \rightarrow B$, and conditions (i)-(iii) hold for $i=0$.

Now assume $i \geq 0$, and that for every $j \leq i$ we have DG algebra homomorphisms $v_{j}: F_{j} \tilde{B} \rightarrow B$ and graded sets $F_{j} X$ satisfying conditions (i)-(iii). We will construct $F_{i+1} \tilde{B}$, etc.

Choose a set $Y_{i+1}^{\prime}$ of elements (of degree $-i-1$ ) and a function $v_{i+1}: Y_{i+1}^{\prime} \rightarrow$ $B^{-i-1}$ such that $\left\{v_{i+1}(y) \mid y \in Y_{i+1}^{\prime}\right\}$ is a set of cocycles that generates $\mathrm{H}^{-i-1} B$ as an $\mathrm{H}^{0} A$-module. For $y \in Y_{i+1}^{\prime}$ define $\mathrm{d}(y):=0$.

Next let

$$
J_{i+1}:=\left\{b \in\left(F_{i} \tilde{B}\right)^{-i} \mid \mathrm{d}(b)=0 \text { and } \mathrm{H}^{-i}\left(v_{i}\right)(b)=0\right\} .
$$

Choose a set $Y_{i+1}^{\prime \prime}$ of elements (of degree $-i-1$ ) and a function $\mathrm{d}: Y_{i+1}^{\prime \prime} \rightarrow J_{i+1}$ such that $\left\{\mathrm{d}(y) \mid y \in Y_{i+1}^{\prime \prime}\right\}$ is a set of elements whose images in $\mathrm{H}^{-i} F_{i} \tilde{B}$ generate $\operatorname{Ker}\left(\mathrm{H}^{-i}\left(v_{i}\right): \mathrm{H}^{-i} F_{i} \tilde{B} \rightarrow \mathrm{H}^{-i} B\right)$ as an $\mathrm{H}^{0} A$-module. Let $y \in Y_{i+1}^{\prime \prime}$. By definition $v_{i}(\mathrm{~d}(y))=\mathrm{d}(b)$ for some $b \in B^{-i}$, and we define $v_{i+1}(y):=b$.

Let $Y_{i+1}:=Y_{i+1}^{\prime} \sqcup Y_{i+1}^{\prime \prime}$ and $F_{i+1} X:=F_{i} X \sqcup Y_{i+1}$. Define the DG algebra $F_{i+1} \tilde{B}$ to be

$$
F_{i+1} \tilde{B}:=F_{i} \tilde{B} \otimes_{\mathbb{Z}} \mathbb{Z}\left[Y_{i+1}\right]
$$

with differential d extending the differential of $F_{i} \tilde{B}$ and the function $\mathrm{d}: Y_{i+1} \rightarrow F_{i} \tilde{B}$ defined above.

(2) This is because at each step in (1) the sets $Y_{i}$ can be chosen to be finite.

(3) Choose elements $b_{1}, \ldots, b_{m} \in B$ that generate it as an $A^{0}$-algebra. Since each $b_{i}$ is integral over $A^{0}$, there is some monic polynomial $p_{i}(y) \in A^{0}[y]$ such that $p_{i}\left(b_{i}\right)=0$. Let $y_{1}, \ldots, y_{m}$ be distinct variables of degree 0 . Define $Y_{0}:=$ $\left\{y_{1}, \ldots, y_{m}\right\}$ and $B^{\dagger}:=A^{0}\left[Y_{0}\right] /\left(p_{1}\left(y_{1}\right), \ldots, p_{m}\left(y_{m}\right)\right)$. This is an $A^{0}$-algebra, which is a free module of finite rank. Let $v_{0}: B^{\dagger} \rightarrow B$ be the surjective $A^{0}$-algebra homomorphism $y_{i} \mapsto b_{i}$. Define $F_{0} \tilde{B}:=A \otimes_{A^{0}} B^{\dagger}$ and $F_{0} X:=\emptyset$. Then conditions (i)-(ii) hold for $i=0$, as well as condition (iii') below.

(iii') $F_{i} \tilde{B}=A\left[Y_{0} \cup F_{i} X\right], \mathrm{d}\left(F_{i} X\right) \subset F_{i-1} \tilde{B}$ and

$$
\text { und } F_{i} \tilde{B} \cong(\text { und } A) \otimes_{A^{0}} A^{0}\left[F_{i} X\right] \otimes_{A^{0}} B^{\dagger} \text {. }
$$

For $i \geq 1$ the proof proceeds as in part (1), but always using condition (iii') instead of (iii).

Proposition 1.8. Suppose we are given three $D G$ algebras $\tilde{A}, \tilde{B}, \tilde{B}^{\prime}$; a ring $B$; and five $D G$ algebra homomorphisms $u, \tilde{u}, \tilde{u}^{\prime}, v, v^{\prime}$ such that the first diagram below is commutative. Assume that $v^{\prime}$ is a quasi-isomorphism, and $\tilde{B}$ is semi-free $D G$ algebra relative to $\tilde{A}$. Then there exists a DG algebra homomorphism $w: \tilde{B} \rightarrow \tilde{B}^{\prime}$ 
such that the second diagram below is commutative:
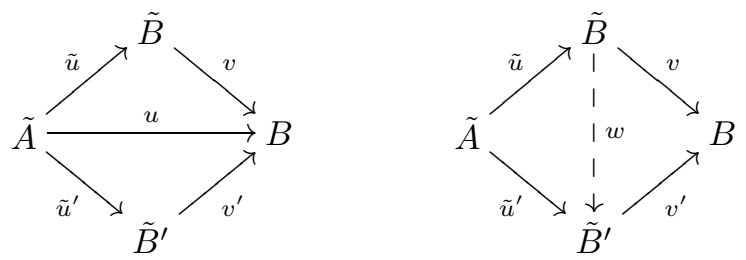

Proof. By definition there is a graded set $X=\coprod_{i \leq 0} X_{i}$ such that

$$
\text { und } \tilde{B} \cong(\text { und } \tilde{A}) \otimes_{\mathbb{Z}} \mathbb{Z}[X] \text {. }
$$

Let's define $F_{i} X:=\bigcup_{j \geq-i} X_{j}$ and $F_{i} \tilde{B}:=\tilde{A}\left[F_{i} X\right] \subset \tilde{B}$. We shall define a compatible sequence of DG algebra homomorphisms $w_{i}: F_{i} \tilde{B} \rightarrow \tilde{B}^{\prime}$, whose union will be called $w$.

For $i=0$ we note that $v^{\prime}: \tilde{B}^{\prime 0} \rightarrow B$ is surjective. Hence there is a function $w_{0}: X_{0} \rightarrow \tilde{B}^{\prime 0}$ such that $v^{\prime}\left(w_{0}(x)\right)=v(x)$ for every $x \in X_{0}$. Since $F_{0} \tilde{B} \cong$ $\tilde{A} \otimes_{\mathbb{Z}} \mathbb{Z}\left[X_{0}\right]$ and $\mathrm{d}\left(w_{0}\left(X_{0}\right)\right)=0$ we can extend the function $w_{0}$ uniquely to a DG algebra homomorphism $w_{0}: F_{0} \tilde{B} \rightarrow \tilde{B}$ such that $w_{0} \circ \tilde{u}=\tilde{u}^{\prime}$.

Now assume that $i \geq 0$ and $w_{i}: F_{i} \tilde{B} \rightarrow \tilde{B}^{\prime}$ has been defined. Let $Y_{i+1}:=$ $F_{i+1} X-F_{i} X$. This is a set of degree $-i-1$ elements. Take any $y \in Y_{i+1}$. Then $\mathrm{d}(y) \in\left(F_{i} \tilde{B}\right)^{-i}$, and we let $b:=w_{i}(\mathrm{~d}(y)) \in \tilde{B}^{\prime-i}$. Because $\mathrm{H} \tilde{B} \cong \mathrm{H} \tilde{B}^{\prime}=B$ there exists an element $c \in \tilde{B}^{\prime-i-1}$ such that $\mathrm{d}(c)=b$. We now define $w_{i+1}(y):=c$. The function $w_{i+1}: Y_{i+1} \rightarrow \tilde{B}^{\prime-i-1}$ extends to a unique DG algebra homomorphism $w_{i+1}: F_{i+1} \tilde{B} \rightarrow \tilde{B}^{\prime}$ such that $\left.w_{i+1}\right|_{F_{i} \tilde{B}}=w_{i}$.

A homomorphism $A \rightarrow A^{\prime}$ between two rings is called a localization if it induces an isomorphism $S^{-1} A \stackrel{\simeq}{\longrightarrow} A^{\prime}$ for some multiplicatively closed subset $S \subset A$. We then say that $A^{\prime}$ is a localization of $A$.

Suppose $A$ is a noetherian ring. An $A$-algebra $B$ is called essentially of finite type if $B$ is a localization of some finitely generated $A$-algebra. Such an algebra $B$ is noetherian. If $C$ is an essentially finite type $B$-algebra, then it is an essentially finite type $A$-algebra.

Proposition 1.9. Let $A$ be a noetherian ring, and let $B$ be an essentially finite type $A$-algebra. Then there is a DG algebra quasi-isomorphism $\tilde{B} \rightarrow B$ such that $\tilde{B}^{0}$ is an essentially finite type $A$-algebra, and each $\tilde{B}^{i}$ is a finitely generated $\tilde{B}^{0}$-module and a flat $A$-module. In particular $\tilde{B}$ is a K-flat $D G A$-module.

Proof. Pick a finitely generated $A$-algebra $B_{\mathrm{f}}$ such that $S^{-1} B_{\mathrm{f}} \cong B$ for some multiplicatively closed subset $S \subset B_{\mathrm{f}}$. According to Proposition 1.7(2) we can find a semi-free DG algebra resolution $\tilde{B}_{\mathrm{f}} \rightarrow B_{\mathrm{f}}$ where $\tilde{B}_{\mathrm{f}}$ has finitely many algebra generators in each degree. Let $\tilde{S} \subset \tilde{B}_{\mathrm{f}}^{0}$ be the pre-image of $S$ under the surjection $\tilde{B}_{\mathrm{f}}^{0} \rightarrow B_{\mathrm{f}}$. Now define $\tilde{B}:=\left(\tilde{S}^{-1} \tilde{B}_{\mathrm{f}}^{0}\right) \otimes_{\tilde{B}_{\mathrm{f}}^{0}} \tilde{B}_{\mathrm{f}}$.

Corollary 1.10. Let $B$ be an essentially finite type $A$-algebra, and let $\tilde{B} \rightarrow B$ be any $K$-flat $D G$ algebra resolution relative to $A$. Then $\mathrm{H}^{0}\left(\tilde{B} \otimes_{A} \tilde{B}\right)$ is an essentially finite type A-algebra, and each $\mathrm{H}^{i}\left(\tilde{B} \otimes_{A} \tilde{B}\right)$ is a finitely generated $\mathrm{H}^{0}\left(\tilde{B} \otimes_{A} \tilde{B}\right)$ module. 
Proof. Using Proposition 1.8, and passing via a semi-free DG algebra resolution, we can replace the given resolution $\tilde{B} \rightarrow B$ by another one satisfying the finiteness conditions in Proposition 1.9. Now the assertion is clear.

Let $M$ be a graded module. The amplitude amp $M \in \mathbb{N} \cup\{\infty\}$ is defined as follows: if $M$ is nonzero and bounded, then

$$
\operatorname{amp} M:=\max \left\{i \in \mathbb{Z} \mid M^{i} \neq 0\right\}-\min \left\{i \in \mathbb{Z} \mid M^{i} \neq 0\right\} .
$$

If $M$ is unbounded, then $\operatorname{amp} M:=\infty$, and if $M=0$, then amp $M:=0$.

Now let $A$ be a DG algebra with $\mathrm{H} A$ bounded, and let $M$ be a DG $A$-module. The flat dimension flat. $\operatorname{dim}_{A} M \in \mathbb{N} \cup\{\infty\}$ is defined as follows:

flat. $\operatorname{dim}_{A} M$

$$
:=\inf \left\{d \in \mathbb{N} \mid \operatorname{ampH}\left(M \otimes_{A}^{\mathrm{L}} N\right) \leq d+\operatorname{ampH} N \text { for all } N \in \operatorname{DGMod} A\right\} .
$$

Observe that $M$ has finite flat dimension if and only if the functor $M \otimes_{A}^{\mathrm{L}}-$ is way out on both sides, in the sense of [RD, Section I.7]. Also, if $M$ has finite flat dimension, then $\mathrm{H} M$ is bounded (take $N:=A$ ). Similarly one can define the projective dimension proj. $\operatorname{dim}_{A} M$ of a DG $A$-module $M$, by considering the amplitude of $\operatorname{HRHom}_{A}(M, N)$. For a ring $A$ and a usual module $M$ the dimensions defined above coincide with the usual ones.

Remark 1.11. Our definition of flat dimension and projective dimension for DG modules differs from that of other authors, e.g. [FIJ].

Proposition 1.12. Let $A$ be a ring, let $B$ and $C$ be $D G A$-algebras, $L \in \mathrm{DGMod} B$, $M \in \operatorname{DGMod} B \otimes_{A} C$ and $N \in \operatorname{DGMod} C$. There exists a functorial morphism

$$
\psi: \operatorname{RHom}_{B}(L, M) \otimes_{C}^{\mathrm{L}} N \rightarrow \operatorname{RHom}_{B}\left(L, M \otimes_{C}^{\mathrm{L}} N\right)
$$

in $\tilde{\mathrm{D}}\left(\mathrm{DGMod} B \otimes_{A} C\right)$. If conditions (i), (ii), and (iii) below hold, then the morphism $\psi$ is an isomorphism.

(i) $\mathrm{H}^{0} B$ is noetherian, $\mathrm{H} L$ is bounded above, and each of the $\mathrm{H}^{0} B$-modules $\mathrm{H}^{i} B$ and $\mathrm{H}^{i} L$ are finitely generated.

(ii) $\mathrm{H} M$ is bounded below, and $\mathrm{H} N$ is bounded.

(iii) Either (a), (b) or (c) is satisfied:

(a) $\mathrm{H}^{i} B=0$ for all $i \neq 0$, and $L$ has finite projective dimension over $B$.

(b) $\mathrm{H}^{i} C=0$ for all $i \neq 0$, and $N$ has finite flat dimension over $C$.

(c) $\mathrm{H}^{i} C=0$ for all $i \neq 0, \mathrm{H}^{0} C$ is noetherian, each $\mathrm{H}^{i} N$ is a finitely generated module over $\mathrm{H}^{0} C$, the canonical morphism $C \rightarrow \operatorname{RHom}_{C}(N, N)$ is an isomorphism, and both $M$ and $\mathrm{RHom}_{B}(L, M)$ have finite flat dimension over $C$.

Proof. The proof is in five steps.

Step 1. To define $\psi$ we may choose a K-projective resolution $P \rightarrow L$ over $B$, and a K-flat resolution $Q \rightarrow N$ over $C$. There is an obvious homomorphism of DG $B \otimes_{A} C$-modules

$$
\psi_{P, Q}: \operatorname{Hom}_{B}(P, M) \otimes_{C} Q \rightarrow \operatorname{Hom}_{B}\left(P, M \otimes_{C} Q\right) .
$$

In the derived category this represents $\psi$.

Step 2. To prove that $\psi$ is an isomorphism (or equivalently that $\psi_{P, Q}$ is a quasiisomorphism) we may forget the $B \otimes_{A} C$-module structures, and consider $\psi$ as a morphism in $\mathrm{D}(\operatorname{Mod} A)$. Now by Proposition $1.4(2)$ we can replace $B$ and $C$ by 
quasi-isomorphic DG $A$-algebras. Thus we may assume both $B$ and $C$ are semi-free as DG $A$-modules.

Step 3. Let's suppose that condition (iii)(a) holds. So $B \rightarrow \mathrm{H}^{0} B$ is a quasiisomorphism. Since $C$ is K-flat over $A$ it follows that $B \otimes_{A} C \rightarrow \mathrm{H}^{0} B \otimes_{A} C$ is also a quasi-isomorphism. By Proposition 1.4 we can assume that $L \in \mathrm{DGMod} \mathrm{H}^{0} B$ and $M \in \mathrm{DGMod}\left(\mathrm{H}^{0} B \otimes_{A} C\right)$, and that $L$ has finite projective dimension over $\mathrm{H}^{0} B$. So we may replace $B$ with $\mathrm{H}^{0} B$, and thus assume that $B$ is a noetherian algebra.

Now choose a resolution $P \rightarrow L$, where $P$ is a bounded complex of finitely generated projective $B$-modules. Take any K-flat resolution $Q \rightarrow N$ over $C$. Then the homomorphism $\psi_{P, Q}$ is actually bijective.

Step 4. Let's assume condition (iii)(b) holds. As in Step 3 we can suppose that $C=C^{0}$. Choose a bounded resolution $Q \rightarrow N$ by flat $C$-modules. By replacing $M$ with the truncation $\tau^{\geq j_{0}} M$ for some $j_{0} \ll 0$, we may assume $M$ is bounded below. According to [AFH, Theorem 9.2.7] we can find a semi-free resolution $P \rightarrow L$ over $B$ such that und $P \cong \bigoplus_{i=-\infty}^{i_{1}}$ und $B[-i]^{\mu_{i}}$ with all the multiplicities $\mu_{i}$ finite. Because the $\mu_{i}$ are finite, $M$ is bounded below and $Q$ is bounded, the homomorphism $\psi_{P, Q}$ is bijective.

Step 5. Finally we consider condition (iii)(c). We can assume that $C=C^{0}$ is noetherian. Since $N \in \mathrm{D}_{\mathrm{f}}^{\mathrm{b}}(\operatorname{Mod} C)$ and $\operatorname{RHom}_{C}(N, N) \cong C$ we see that the support of $N$ is $\operatorname{Spec} C$. By Lemma 1.13 below we conclude that $N$ generates $\mathrm{D}^{+}(\operatorname{Mod} C)$. Let

$$
\psi^{\prime}: \operatorname{RHom}_{C}\left(N, \operatorname{RHom}_{B}(L, M) \otimes_{C}^{\mathrm{L}} N\right) \rightarrow \operatorname{RHom}_{C}\left(N, \operatorname{RHom}_{B}\left(L, M \otimes_{C}^{\mathrm{L}} N\right)\right)
$$

be the morphism obtained from $\psi$ by applying the functor $\operatorname{RHom}_{C}(N,-)$. Since $\psi$ is a morphism in $\mathrm{D}^{+}(\operatorname{Mod} C)$, in order to prove it is an isomorphism it suffices to prove that $\psi^{\prime}$ is an isomorphism.

Consider the commutative diagram of morphisms in $\mathrm{D}(\operatorname{Mod} C)$

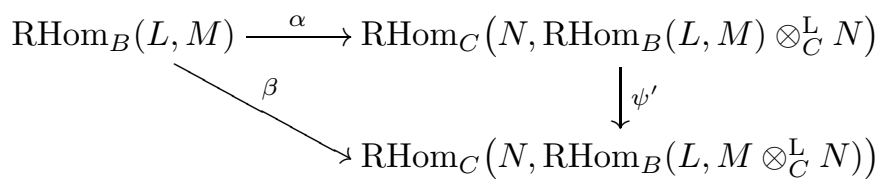

in which $\alpha$ and $\beta$ are the obvious morphisms. We shall prove that $\alpha$ and $\beta$ are isomorphisms. The complex $\operatorname{RHom}_{B}(L, M)$ has finite flat dimension over $C$, so using the proposition with condition (iii)(b), which we already proved, we have

$$
\begin{aligned}
\operatorname{RHom}_{C}\left(N, \operatorname{RHom}_{B}(L, M) \otimes_{C}^{\mathrm{L}} N\right) & \cong \operatorname{RHom}_{C}(N, N) \otimes_{C}^{\mathrm{L}} \operatorname{RHom}_{B}(L, M) \\
& \cong \operatorname{RHom}_{B}(L, M) .
\end{aligned}
$$

But the composed isomorphism is precisely $\alpha$. On the other hand the complex $M$ has finite flat dimension over $C$, so using the proposition with condition (iii)(b) once more (for the isomorphism marked $\diamond$ ), we have

$$
\begin{aligned}
& \operatorname{RHom}_{C}\left(N, \operatorname{RHom}_{B}\left(L, M \otimes_{C}^{\mathrm{L}} N\right)\right) \cong \operatorname{RHom}_{B}\left(L, \operatorname{RHom}_{C}\left(N, M \otimes_{C}^{\mathrm{L}} N\right)\right) \\
& \quad \cong \operatorname{RHom}_{B}\left(L, M \otimes_{C}^{\mathrm{L}} \operatorname{RHom}_{C}(N, N)\right) \cong \operatorname{RHom}_{B}(L, M) .
\end{aligned}
$$

Here the composed isomorphism is $\beta$.

Let $C$ be a noetherian ring. Recall that given a complex $N \in \mathrm{D}_{\mathrm{f}}^{\mathrm{b}}(\operatorname{Mod} C)$ its support is defined to be $\bigcup_{i} \operatorname{Supp} \mathrm{H}^{i} N \subset \operatorname{Spec} C$. The complex $N$ is said to generate $\mathrm{D}^{+}(\operatorname{Mod} C)$ if for any nonzero object $M \in \mathrm{D}^{+}(\operatorname{Mod} C)$ one has $\operatorname{RHom}_{C}(N, M) \neq 0$. 
Lemma 1.13. Suppose $C$ is a noetherian ring and $N \in \mathrm{D}_{\mathrm{f}}^{\mathrm{b}}(\operatorname{Mod} C)$ is a complex whose support is $\operatorname{Spec} C$. Then $N$ generates $\mathrm{D}^{+}(\operatorname{Mod} C)$.

Proof. Suppose $M$ is a nonzero object in $\mathrm{D}^{+}(\operatorname{Mod} C)$. We have to prove that $\operatorname{RHom}_{C}(N, M) \neq 0$. Let $i_{0}:=\min \left\{i \in \mathbb{Z} \mid \mathrm{H}^{i} M \neq 0\right\}$, and choose a nonzero finitely generated submodule $M^{\prime} \subset \mathrm{H}^{i_{0}} M$. Let $\mathfrak{p}$ be a minimal prime ideal in the support of $M^{\prime}$, so that $M_{\mathfrak{p}}^{\prime}:=B_{\mathfrak{p}} \otimes_{C} M^{\prime}$ is a nonzero finite length module over the local ring $C_{\mathfrak{p}}$. Now $N_{\mathfrak{p}}$ is a nonzero object of $\mathrm{D}_{\mathfrak{f}}^{\mathrm{b}}\left(\operatorname{Mod} C_{\mathfrak{p}}\right)$. Let $j_{1}:=\max \left\{j \in \mathbb{Z} \mid \mathrm{H}^{j} N_{\mathfrak{p}} \neq 0\right\}$. Since $\mathrm{H}^{j_{1}} N_{\mathfrak{p}}$ is a nonzero finitely generated $B_{\mathfrak{p}}$-module, there exists a nonzero homomorphism $\phi: \mathrm{H}^{j_{1}} N_{\mathfrak{p}} \rightarrow M_{\mathfrak{p}}^{\prime}$. This $\phi$ can be interpreted as a nonzero element of $\operatorname{Ext}_{C_{\mathfrak{p}}}^{i_{0}-j_{1}}\left(N_{\mathfrak{p}}, M_{\mathfrak{p}}\right)$.

Finally, by Proposition 1.12 under its condition (iii)(b) - whose proof does not rely on this lemma - we have an isomorphism

$$
\operatorname{Ext}_{C_{\mathfrak{p}}}^{i_{0}-j_{1}}\left(N_{\mathfrak{p}}, M_{\mathfrak{p}}\right) \cong C_{\mathfrak{p}} \otimes_{C} \operatorname{Ext}_{C}^{i_{0}-j_{1}}(N, M) \text {. }
$$

Remark 1.14. Assume $A$ is noetherian. Proposition 1.12 can be extended by replacing conditions (iii)(a) and (iii)(b) respectively with: (iii) $\left(\mathrm{a}^{\prime}\right) \mathrm{H} B$ is a bounded essentially finite type $A$-algebra, and $L$ has finite projective dimension over $B$; and (iii) ( $\left.\mathrm{b}^{\prime}\right) \mathrm{HC}$ is a bounded essentially finite type $A$-algebra, $\mathrm{H} N$ is a finitely generated $\mathrm{H} C$-module, and $N$ has finite flat dimension over $B$. The trick for (iii) $\left(\mathrm{a}^{\prime}\right)$ is to localize on Spec $\mathrm{H}^{0} B$ and to look at minimal semi-free resolutions of $L$. This trick also shows that flat.dim $B=$ proj. $\operatorname{dim}_{B} L$. Details will appear elsewhere.

\section{THE SQUARING OPERATION}

In this section we introduce a key technical notion used in the definition of rigidity, namely the squaring operation. This operation is easy to define when the base ring is a field (see Corollary 2.8), but otherwise there are complications, due to torsion. We solve the problem using DG algebras.

Recall that for a DG algebra $A$ the derived category of DG modules is denoted by $\tilde{\mathrm{D}}(\operatorname{DGMod} A)$. If $A$ is a ring, then $\tilde{\mathrm{D}}(\operatorname{DGMod} A)=\mathrm{D}(\operatorname{Mod} A)$.

Let $A$ be a ring, and let $M \in \mathrm{D}(\operatorname{Mod} A)$. As explained earlier the derived tensor product $M \otimes_{A}^{\mathrm{L}} M \in \mathrm{D}(\operatorname{Mod} A)$ is defined to be $M \otimes_{A}^{\mathrm{L}} M:=\tilde{M} \otimes_{A} \tilde{M}$, where $\tilde{M} \rightarrow M$ is any $\mathrm{K}$-flat resolution of $M$. If $M \in \mathrm{DGMod} B$ for some DG $A$-algebra $B$, then we would like to be able to make $M \otimes_{A}^{\mathrm{L}} M$ into an object of $\tilde{\mathrm{D}}\left(\mathrm{DGMod} B \otimes_{A} B\right)$. But this is not always possible, at least not in any obvious way, due to torsion. (For instance take $A:=\mathbb{Z}$ and $M=B:=\mathbb{Z} /(2))$. Fortunately there is a way to get around this problem.

Recall that a semi-free DG $A$-algebra $\tilde{B}$ is also semi-free as a DG $A$-module, and hence it is a K-flat DG $A$-module. Thus Proposition 1.7 implies that there exist K-flat DG algebra resolutions $A \rightarrow \tilde{B} \rightarrow B$ of $A \rightarrow B$.

Lemma 2.1. Let $A$ be a ring and $B$ a $D G$ A-algebra. Suppose $A \rightarrow \tilde{B} \rightarrow B$ is any $K$-flat $D G$ algebra resolution of $A \rightarrow B$. Then the (nonadditive) functor

$$
\tilde{\mathrm{D}}(\mathrm{DGMod} B) \rightarrow \mathrm{D}(\operatorname{Mod} A), M \mapsto M \otimes_{A}^{\mathrm{L}} M,
$$

factors canonically through $\tilde{\mathrm{D}}\left(\mathrm{DGMod} \tilde{B} \otimes_{A} \tilde{B}\right)$. 
Proof. Choose any quasi-isomorphism $\tilde{M} \rightarrow M$ in DGMod $\tilde{B}$ with $\tilde{M}$ K-flat over $A$. This is possible since any K-flat DG $\tilde{B}$-module is K-flat over $A$. We get $M \otimes_{A}^{\mathrm{L}} M=$ $\tilde{M} \otimes_{A} \tilde{M} \in \tilde{\mathrm{D}}\left(\mathrm{DGMod} \tilde{B} \otimes_{A} \tilde{B}\right)$.

Theorem 2.2. Let $A$ be a ring, let $B$ be an $A$-algebra and let $M$ be a $D G B$ module. Choose a K-flat DG algebra resolution $A \rightarrow \tilde{B} \rightarrow B$ of $A \rightarrow B$. Then the object

$$
\mathrm{Sq}_{B / A} M:=\operatorname{RHom}_{\tilde{B} \otimes_{A} \tilde{B}}\left(B, M \otimes_{A}^{\mathrm{L}} M\right) \in \mathrm{D}(\operatorname{Mod} B),
$$

where the $B$-module structure is via the action on the first argument of RHom, is independent of the choice of resolution $A \rightarrow \tilde{B} \rightarrow B$.

The functorial property of the assignment $M \mapsto \mathrm{Sq}_{B / A} M$ is stated in Theorem 2.3 below.

Proof. The idea for the proof was communicated to us by Bernhard Keller. Choose some semi-free DG algebra resolution $A \rightarrow \tilde{B}^{\prime} \rightarrow B$ of $A \rightarrow B$ (see Proposition 1.7). We will show that there is a canonical isomorphism

$$
\chi: \operatorname{RHom}_{\tilde{B} \otimes_{A} \tilde{B}}\left(B, M \otimes_{A}^{\mathrm{L}} M\right) \stackrel{\simeq}{\longrightarrow} \operatorname{RHom}_{\tilde{B}^{\prime} \otimes_{A} \tilde{B}^{\prime}}\left(B, M \otimes_{A}^{\mathrm{L}} M\right)
$$

in $\mathrm{D}(\operatorname{Mod} B)$.

Let us choose a K-projective resolution $\tilde{M} \rightarrow M$ over $\tilde{B}$, and a K-injective resolution $\tilde{M} \otimes_{A} \tilde{M} \rightarrow \tilde{I}$ over $\tilde{B} \otimes_{A} \tilde{B}$. So

$$
\operatorname{RHom}_{\tilde{B} \otimes_{A} \tilde{B}}\left(B, M \otimes_{A}^{\mathrm{L}} M\right)=\operatorname{Hom}_{\tilde{B} \otimes_{A} \tilde{B}}(B, \tilde{I}) .
$$

Likewise let's choose resolutions $\tilde{M}^{\prime} \rightarrow M$ and $\tilde{M}^{\prime} \otimes_{A} \tilde{M}^{\prime} \rightarrow \tilde{I}^{\prime}$ over $\tilde{B}^{\prime}$ and $\tilde{B}^{\prime} \otimes_{A} \tilde{B}^{\prime}$, respectively.

By Proposition 1.8 there is a DG algebra quasi-isomorphism $u_{0}: \tilde{B}^{\prime} \rightarrow \tilde{B}$ that's compatible with the quasi-isomorphisms to $B$. By the categorical properties of $K$-projective resolutions there is an $\tilde{B}^{\prime}$-linear quasi-isomorphism $\phi_{0}: \tilde{M}^{\prime} \rightarrow \tilde{M}$, that's compatible up to homotopy with the quasi-isomorphisms to $M$. We obtain an $\tilde{B}^{\prime} \otimes_{A} \tilde{B}^{\prime}$-linear quasi-isomorphism $\phi_{0} \otimes \phi_{0}: \tilde{M}^{\prime} \otimes_{A} \tilde{M}^{\prime} \rightarrow \tilde{M} \otimes_{A} \tilde{M}$. Next by the categorical properties of $K$-injective resolutions there is a $\tilde{B}^{\prime} \otimes_{A} \tilde{B}^{\prime}$-linear quasi-isomorphism $\psi_{0}: \tilde{I} \rightarrow \tilde{I}^{\prime}$ that's compatible up to homotopy with the quasiisomorphisms from $\tilde{M}^{\prime} \otimes_{A} \tilde{M}^{\prime}$. We thus get a $B$-linear homomorphism

$$
\chi_{0}: \operatorname{Hom}_{\tilde{B} \otimes_{A} \tilde{B}}(B, \tilde{I}) \rightarrow \operatorname{Hom}_{\tilde{B}^{\prime} \otimes_{A} \tilde{B}^{\prime}}\left(B, \tilde{I}^{\prime}\right) .
$$

Proposition 1.4 shows that $\chi_{0}$ is in fact an isomorphism in $\mathrm{D}(\operatorname{Mod} B)$.

Now suppose $u_{1}: \tilde{B}^{\prime} \rightarrow \tilde{B}, \phi_{1}: \tilde{M}^{\prime} \rightarrow \tilde{M}$ and $\psi_{1}: \tilde{I} \rightarrow \tilde{I}^{\prime}$ are other choices of quasi-isomorphisms of the same respective types as $u_{0}, \phi_{0}$ and $\psi_{0}$. Then we get an induced isomorphism

$$
\chi_{1}: \operatorname{Hom}_{\tilde{B} \otimes_{A} \tilde{B}}(B, \tilde{I}) \rightarrow \operatorname{Hom}_{\tilde{B}^{\prime} \otimes_{A} \tilde{B}^{\prime}}\left(B, \tilde{I}^{\prime}\right)
$$

in $\mathrm{D}(\operatorname{Mod} B)$. We shall prove that $\chi_{1}=\chi_{0}$.

Here we have to introduce an auxiliary DG $\tilde{B}$-module $\mathrm{C}(\tilde{M})$, the cylinder module. As a graded module one has

$$
\mathrm{C}(\tilde{M}):=\left[\begin{array}{cc}
\tilde{M} & \tilde{M}[-1] \\
0 & \tilde{M}
\end{array}\right]
$$


a triangular matrix module, and the differential is

$$
\mathrm{d}\left(\left[\begin{array}{cc}
m_{0} & n \\
0 & m_{1}
\end{array}\right]\right):=\left[\begin{array}{cc}
\mathrm{d}\left(m_{0}\right) & m_{0}-m_{1}-\mathrm{d}(n) \\
0 & \mathrm{~d}\left(m_{1}\right)
\end{array}\right]
$$

for $m_{0}, m_{1}, n \in \tilde{M}$. There are DG module quasi-isomorphisms $\epsilon: \tilde{M} \rightarrow \mathrm{C}(\tilde{M})$ and $\eta_{0}, \eta_{1}: \mathrm{C}(\tilde{M}) \rightarrow \tilde{M}$, with formulas

$$
\epsilon(m):=\left[\begin{array}{cc}
m & 0 \\
0 & m
\end{array}\right] \text { and } \eta_{i}\left(\left[\begin{array}{cc}
m_{0} & n \\
0 & m_{1}
\end{array}\right]\right):=m_{i} .
$$

The cylinder module $\mathrm{C}(\tilde{M})$ is a DG module over $\tilde{B}$ by the formula

$$
b \cdot\left[\begin{array}{cc}
m_{0} & n \\
0 & m_{1}
\end{array}\right]:=\left[\begin{array}{cc}
b m_{0} & b n \\
0 & b m_{1}
\end{array}\right] .
$$

There is a quasi-isomorphism of DG $\tilde{B}$-modules

$$
\mathrm{C}(\tilde{M}) \rightarrow\left[\begin{array}{cc}
\tilde{M} & M[-1] \\
0 & \tilde{M}
\end{array}\right]
$$

which is the identity on the diagonal elements, and the given quasi-isomorphism $\tilde{M} \rightarrow M$ in the corner. The two $\tilde{B}^{\prime}$-linear quasi-isomorphisms $\phi_{0}$ and $\phi_{1}$ fit into a $\tilde{B}^{\prime}$-linear quasi-isomorphism

$$
\tilde{M}^{\prime} \stackrel{\left[\begin{array}{cc}
\phi_{0} & 0 \\
0 & \phi_{1}
\end{array}\right]}{\longrightarrow}\left[\begin{array}{cc}
\tilde{M} & M[-1] \\
0 & \tilde{M}
\end{array}\right] .
$$

Since $\tilde{M}^{\prime}$ is K-projective over $\tilde{B}^{\prime}$ we can lift $\left[\begin{array}{cc}\phi_{0} & 0 \\ 0 & \phi_{1}\end{array}\right]$ to a quasi-isomorphism $\phi$ : $\tilde{M}^{\prime} \rightarrow \mathrm{C}(\tilde{M})$ such that $\eta_{i} \circ \phi=\phi_{i}$ up to homotopy.

Let's choose a K-injective resolution $\mathrm{C}(\tilde{M}) \otimes_{A} \mathrm{C}(\tilde{M}) \rightarrow \tilde{K}$ over $\tilde{B} \otimes_{A} \tilde{B}$. Then for $i=0,1$ we have a diagram

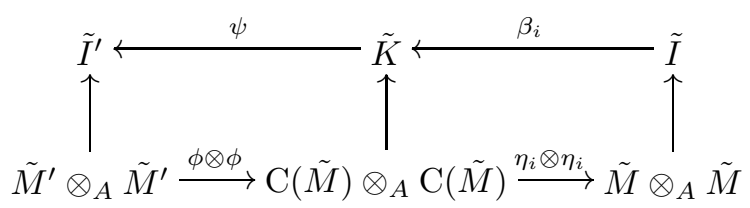

that's commutative up to homotopy. Here $\psi$ and $\beta_{i}$ are some DG module homomorphisms, which exist due to the K-injectivity of $\tilde{I}^{\prime}$ and $\tilde{K}$, respectively. Because $\phi_{i} \otimes \phi_{i}=\left(\eta_{i} \otimes \eta_{i}\right) \circ(\phi \otimes \phi)$ up to homotopy, and $\tilde{I}^{\prime}$ is K-injective, it follows that the $\tilde{B}^{\prime} \otimes_{A} \tilde{B}^{\prime}$-linear DG module quasi-isomorphisms $\psi \circ \beta_{i}$ and $\psi_{i}$ are homotopic. Therefore in order to prove that $\chi_{0}=\chi_{1}$ it suffices to prove that the two isomorphisms in $\mathrm{D}(\operatorname{Mod} B)$

$$
\theta_{0}, \theta_{1}: \operatorname{Hom}_{\tilde{B} \otimes_{A} \tilde{B}}(B, \tilde{I}) \rightarrow \operatorname{Hom}_{\tilde{B} \otimes_{A} \tilde{B}}(B, \tilde{K}),
$$

that are induced by $\beta_{0}, \beta_{1}$, respectively, are equal.

For $i=0,1$ consider the diagram

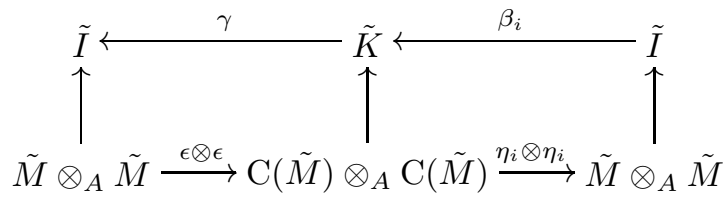


where $\gamma$ is some $\tilde{B} \otimes_{A} \tilde{B}$-linear DG module homomorphism, chosen so as to make the left square commute up to homotopy. As before, since $\left(\eta_{i} \otimes \eta_{i}\right) \circ(\epsilon \otimes \epsilon)=\mathbf{1}_{\tilde{M} \otimes_{A}} \tilde{M}$ it follows that $\gamma \circ \beta_{i}$ and $\mathbf{1}_{\tilde{I}}$ are homotopic. Hence both $\theta_{0}$ and $\theta_{1}$ are inverses of the isomorphism

in $\mathrm{D}(\operatorname{Mod} B)$ induced by $\gamma$, so $\theta_{0}=\theta_{1}$.

$$
\operatorname{Hom}_{\tilde{B} \otimes_{A} \tilde{B}}(B, \tilde{K}) \stackrel{\simeq}{\longrightarrow} \operatorname{Hom}_{\tilde{B} \otimes_{A} \tilde{B}}(B, \tilde{I})
$$

Suppose we are given a DG algebra homomorphism $B_{1} \rightarrow B_{2}$; objects $L_{i}, M_{i} \in$ DGMod $B_{i}$; and homomorphisms $\psi: L_{2} \rightarrow L_{1}$ and $\phi: M_{1} \rightarrow M_{2}$ in DGMod $B_{1}$. We then denote by

$$
(\psi, \phi): \operatorname{Hom}_{B_{1}}\left(L_{1}, M_{1}\right) \rightarrow \operatorname{Hom}_{B_{2}}\left(L_{2}, M_{2}\right)
$$

the homomorphism $\chi \mapsto \phi \circ \chi \circ \psi$. The same notation will be used for morphisms in derived categories.

Theorem 2.3. Let $A$ be a ring, let $B$ and $C$ be $A$-algebras, and let $M \in \mathrm{D}(\operatorname{Mod} B)$ and $N \in \mathrm{D}(\operatorname{Mod} C)$. Let $u: B \rightarrow C$ be an $A$-algebra homomorphism, and let $\phi: N \rightarrow M$ be a morphism in $\mathrm{D}(\operatorname{Mod} B)$. Suppose $A \rightarrow \tilde{B} \rightarrow B$ is a K-projective $D G$ algebra resolution of $A \rightarrow B$, and $\tilde{B} \rightarrow \tilde{C} \rightarrow C$ is a K-projective DG algebra resolution of $\tilde{B} \rightarrow C$. Define a morphism

$$
\mathrm{Sq}_{u / A}(\phi): \mathrm{Sq}_{C / A} N \rightarrow \mathrm{Sq}_{B / A} M
$$

in $\mathrm{D}(\operatorname{Mod} B)$ by the formula

$$
\begin{aligned}
\operatorname{Sq}_{u / A}(\phi):=(u, \phi \otimes \phi): \operatorname{RHom}_{\tilde{C} \otimes_{A} \tilde{C}}\left(C, N \otimes_{A}^{\mathrm{L}} N\right) & \\
& \rightarrow \operatorname{RHom}_{\tilde{B} \otimes_{A} \tilde{B}}\left(B, M \otimes_{A}^{\mathrm{L}} M\right) .
\end{aligned}
$$

Then the morphism $\mathrm{Sq}_{u / A}(\phi)$ is independent of the resolutions $A \rightarrow \tilde{B} \rightarrow B$ and $\tilde{B} \rightarrow \tilde{C} \rightarrow C$.

The existence of these DG algebra resolutions is guaranteed by Propositions 1.7 and 1.8 .

Proof. Let's start by making the morphism $(u, \phi \otimes \phi)$ explicit. Note that $\tilde{C}$ is also K-projective relative to $A$, so it may be used to calculate $\mathrm{Sq}_{C / A} N$. Let's choose DG module resolutions $\tilde{M} \rightarrow M, \tilde{N} \rightarrow N, \tilde{M} \otimes_{A} \tilde{M} \rightarrow \tilde{I}$ and $\tilde{N} \otimes_{A} \tilde{N} \rightarrow \tilde{J}$ by $\mathrm{K}$-projective or K-injective DG modules over the appropriate DG algebras, as was done in the proof of Theorem 2.2. Since $\tilde{N}$ is a K-projective DG $\tilde{B}$-module we get an actual DG module homomorphism $\tilde{\phi}: \tilde{N} \rightarrow \tilde{M}$ representing $\phi$. Therefore there is a $\tilde{B} \otimes_{A} \tilde{B}$-linear DG module homomorphism $\tilde{\phi} \otimes \tilde{\phi}: \tilde{N} \otimes_{A} \tilde{N} \rightarrow \tilde{M} \otimes_{A} \tilde{M}$. Because $\tilde{I}$ is K-injective we obtain a DG module homomorphism $\psi: \tilde{J} \rightarrow \tilde{I}$ lifting $\tilde{N} \otimes_{A} \tilde{N} \stackrel{\tilde{\phi} \otimes \tilde{\phi}}{\longrightarrow} \tilde{M} \otimes_{A} \tilde{M} \rightarrow \tilde{I}$. In this way we have obtained a homomorphism

$$
(u, \psi): \operatorname{Hom}_{\tilde{C} \otimes_{A} \tilde{C}}(C, \tilde{J}) \rightarrow \operatorname{Hom}_{\tilde{B} \otimes_{A} \tilde{B}}(B, \tilde{I})
$$

in $\mathrm{DGMod} B$, which represents $\mathrm{Sq}_{u / A}(\phi)$ once we pass to $\mathrm{D}(\operatorname{Mod} B)$.

Any other choices of K-projective and K-injective DG module resolutions, and of the DG module homomorphisms $\tilde{\phi}$ and $\psi$, would yield a homomorphism that is homotopy equivalent to $(u, \psi)$. So the resulting morphism $\mathrm{Sq}_{u / A}(\phi)$ in $\mathrm{D}(\operatorname{Mod} B)$ will be unchanged.

It remains to prove $\mathrm{Sq}_{u / A}(\phi)$ is independent of the choice of DG algebra resolutions. Suppose we choose a semi-free DG algebra resolution $A \rightarrow \tilde{B}^{\prime} \rightarrow B$ of 
$A \rightarrow B$, and a semi-free DG algebra resolution $\tilde{B}^{\prime} \stackrel{\tilde{u}^{\prime}}{\rightarrow} \tilde{C}^{\prime} \rightarrow C$ of $\tilde{B}^{\prime} \rightarrow C$. After choosing DG module resolutions $\tilde{M}^{\prime} \rightarrow M, \tilde{N}^{\prime} \rightarrow N, \tilde{M}^{\prime} \otimes_{A} \tilde{M}^{\prime} \rightarrow \tilde{I}^{\prime}$ and $\tilde{N}^{\prime} \otimes_{A} \tilde{N}^{\prime} \rightarrow \tilde{J}^{\prime}$ by K-projective or K-injective DG modules over the appropriate DG algebras, we obtain a homomorphism $\psi^{\prime}: \tilde{J}^{\prime} \rightarrow \tilde{I}^{\prime}$ of DG $\tilde{B}^{\prime} \otimes_{A} \tilde{B}^{\prime}$-modules, and a homomorphism

$$
\operatorname{Sq}_{u / A}^{\prime}(\phi):=\left(u, \psi^{\prime}\right): \operatorname{Hom}_{\tilde{C}^{\prime} \otimes_{A} \tilde{C}^{\prime}}\left(C, \tilde{J}^{\prime}\right) \rightarrow \operatorname{Hom}_{\tilde{B}^{\prime} \otimes_{A} \tilde{B}^{\prime}}\left(B, \tilde{I}^{\prime}\right)
$$

in DGMod $B$.

Applying Proposition 1.8 twice we can find DG algebra homomorphisms $v_{0}$ and $w_{0}$ such that the diagram of DG algebra homomorphisms

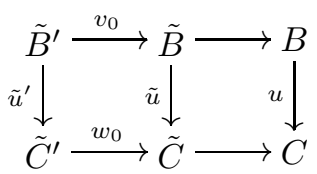

is commutative. As in the proof of Theorem 2.2 we pick quasi-isomorphisms $\psi_{M, 0}$ : $\tilde{I} \rightarrow \tilde{I}^{\prime}$ and $\psi_{N, 0}: \tilde{J} \rightarrow \tilde{J}^{\prime}$ over $\tilde{B}^{\prime} \otimes_{A} \tilde{B}^{\prime}$ and $\tilde{C}^{\prime} \otimes_{A} \tilde{C}^{\prime}$, respectively. Then we get a commutative up to homotopy diagram

$$
\begin{aligned}
& \operatorname{Hom}_{\tilde{B} \otimes_{A} \tilde{B}}(B, \tilde{I}) \stackrel{\chi_{M, 0}}{\longrightarrow} \operatorname{Hom}_{\tilde{B}^{\prime} \otimes_{A} \tilde{B}^{\prime}}\left(B, \tilde{I}^{\prime}\right) \\
& \operatorname{Sq}_{u / A}(\phi) \uparrow \\
& \operatorname{Hom}_{\tilde{C} \otimes_{A} \tilde{C}}(C, \tilde{J}) \stackrel{\chi_{N, 0}^{\prime}}{\longrightarrow} \operatorname{Hom}_{\tilde{C}_{u / A}^{\prime} \otimes_{A}(\phi)}\left(C, \tilde{C}^{\prime}\right. \\
&
\end{aligned}
$$

in DGMod $B$, where the horizontal arrows are quasi-isomorphisms. If we were to choose another pair of DG algebra quasi-isomorphisms $v_{1}: \tilde{B}^{\prime} \rightarrow \tilde{B}$ and $w_{1}: \tilde{C}^{\prime} \rightarrow$ $\tilde{C}$ so as to make diagram (2.4) commutative, then according to Theorem 2.2 there would be equalities $\chi_{M, 0}=\chi_{M, 1}$ and $\chi_{N, 0}=\chi_{N, 1}$ of isomorphisms in $\mathrm{D}(\operatorname{Mod} B)$. Therefore $\mathrm{Sq}_{u / A}^{\prime}(\phi)=\mathrm{Sq}_{u / A}(\phi)$ as morphisms in $\mathrm{D}(\operatorname{Mod} B)$.

Corollary 2.5. Let $A$ be a ring, let $B, C, D$ be $A$-algebras, and let $M \in \mathrm{D}(\operatorname{Mod} B)$, $N \in \mathrm{D}(\operatorname{Mod} C)$ and $P \in \mathrm{D}(\operatorname{Mod} D)$. Let $B \stackrel{u}{\rightarrow} C \stackrel{v}{\rightarrow} D$ be A-algebra homomorphisms, let $\phi: N \rightarrow M$ be a morphism in $\mathrm{D}(\operatorname{Mod} B)$, and let $\psi: P \rightarrow N$ be a morphism in $\mathrm{D}(\operatorname{Mod} C)$.

(1) There is equality

$$
\mathrm{Sq}_{v \circ u / A}(\phi \circ \psi)=\mathrm{Sq}_{u / A}(\phi) \circ \mathrm{Sq}_{v / A}(\psi)
$$

in $\operatorname{Hom}_{\mathrm{D}(\operatorname{Mod} B)}\left(\mathrm{Sq}_{D / A} P, \mathrm{Sq}_{B / A} M\right)$.

(2) If $B=C, M=N, u=\mathbf{1}_{B}$ and $\phi=\mathbf{1}_{M}$ (the identity morphisms), then $\mathrm{Sq}_{u / A}(\phi)=\mathbf{1}_{\mathrm{Sq}_{B / A} M}$.

Proof. (1) Just choose a K-projective DG algebra resolution $\tilde{C} \rightarrow \tilde{D} \rightarrow D$ of $\tilde{C} \rightarrow D$, and use the fact that

$$
(v \circ u,(\phi \circ \psi) \otimes(\phi \circ \psi))=(u, \phi \otimes \phi) \circ(v, \psi \otimes \psi) .
$$

(2) Clear from the definitions.

For the identity homomorphism $\mathbf{1}_{B}: B \rightarrow B$ we write $\mathrm{Sq}_{B / A}(\phi):=\operatorname{Sq}_{1_{B} / A}(\phi)$. 
Definition 2.6. Let $A$ be a ring and let $B$ be an $A$-algebra. The (nonlinear) functor

$$
\mathrm{Sq}_{B / A}: \mathrm{D}(\operatorname{Mod} B) \rightarrow \mathrm{D}(\operatorname{Mod} B)
$$

from Theorems 2.2 and 2.3 is called the squaring operation over $B$ relative to $A$.

The next result explains the name "squaring".

Corollary 2.7. In the situation of Theorem 2.3 let $c \in C$. Then

$$
\mathrm{Sq}_{u / A}(c \phi)=c^{2} \mathrm{Sq}_{u / A}(\phi) .
$$

Proof. It suffices to consider $u=\mathbf{1}_{C}: C \rightarrow C$ and $\phi=\mathbf{1}_{N}: N \rightarrow N$. Choose any lifting of $c$ to $\tilde{c} \in \tilde{C}^{0}$. Then multiplication by $\tilde{c} \otimes \tilde{c}$ on $\tilde{J}$ has the same effect on $\operatorname{Hom}_{\tilde{C} \otimes_{A} \tilde{C}}(C, \tilde{J})$, up to homotopy, as multiplication by $c^{2}$ on $C$.

Corollary 2.8. Suppose $B$ is a flat A-algebra, and $M$ is a bounded above complex of $B$-modules that are flat as $A$-modules. Then there is a functorial isomorphism

$$
\mathrm{Sq}_{B / A} M \cong \operatorname{RHom}_{B \otimes_{A} B}\left(B, M \otimes_{A} M\right) .
$$

Proof. This is because $B$ and $M$ are K-flat DG $A$-modules.

Remark 2.9. One might be tempted to use the notation $\operatorname{RHom}_{B \otimes{ }_{A} B}\left(B, M \otimes_{A}^{\mathrm{L}} M\right)$ instead of $\mathrm{Sq}_{B / A} M$. Indeed it is possible to make sense of the "DG algebra" $B \otimes_{A}^{\mathrm{L}} B$, as an object of a suitable Quillen localization of the category of DG $A$ algebras. Cf. [Hi] and [Ta], and also [Qu], where an analogous construction was made using simplicial algebras rather than DG algebras. Then one should show that the triangulated category " $\tilde{D}\left(\operatorname{DGMod} B \otimes_{A}^{\mathrm{L}} B\right)$ " is well-defined, etc. See also [Dr, Appendix V].

\section{EsSentially SMOOTH HOMOMORPHISMS}

From here on all rings are by default noetherian. We shall use notation such as $f^{*}: A \rightarrow B$ for a ring homomorphism; so that $f: \operatorname{Spec} B \rightarrow \operatorname{Spec} A$ is the corresponding morphism of schemes. This will make our notation for various functors more uniform. For instance restriction of scalars becomes $f_{*}: \operatorname{Mod} B \rightarrow \operatorname{Mod} A$, and extension of scalars (i.e. $M \mapsto B \otimes_{A} M$ ) becomes $f^{*}: \operatorname{Mod} A \rightarrow \operatorname{Mod} B$. See also Definitions 5.1 and 6.2. Given another algebra homomorphism $g^{*}: B \rightarrow C$ we shall sometimes write $(f \circ g)^{*}:=g^{*} \circ f^{*}$.

In this section we present some results in commutative algebra. Recall that an $A$-algebra $B$ is called formally smooth (resp. formally étale) if it has the lifting property (resp. the unique lifting property) for infinitesimal extensions. The $A$ algebra $B$ is called smooth (resp. étale) if it is finitely generated and formally smooth (resp. formally étale). If $B$ is smooth over $A$, then it is flat, and $\Omega_{B / A}^{1}$ is a finitely generated projective $B$-module. See [EGA, Section $0_{\mathrm{IV}} \cdot 19.3$ ] and [EGA, Section IV.17.3] for details.

Definition 3.1. Let $A$ and $B$ be noetherian rings. A ring homomorphism $f^{*}$ : $A \rightarrow B$ is called essentially smooth (resp. essentially étale) if it is of essentially finite type and formally smooth (resp. formally étale). In this case $B$ is called an essentially smooth (resp. essentially étale) $A$-algebra.

Observe that smooth homomorphisms and localizations are essentially smooth. 
Proposition 3.2. Let $f^{*}: A \rightarrow B$ be an essentially smooth homomorphism.

(1) There is an open covering $\operatorname{Spec} B=\bigcup_{i} \operatorname{Spec} B_{i}$ such that for every $i$ the homomorphism $A \rightarrow B_{i}$ is the composition of a smooth homomorphism $A \rightarrow B_{i}^{\mathrm{sm}}$ and a localization $B_{i}^{\mathrm{sm}} \rightarrow B_{i}$.

(2) $f^{*}$ is flat, and $\Omega_{B / A}^{1}$ is a finitely generated projective $B$-module.

(3) $f^{*}$ is essentially étale if and only if $\Omega_{B / A}^{1}=0$.

(4) Let $g^{*}: B \rightarrow C$ be another essentially smooth homomorphism. Then $g^{*} \circ$ $f^{*}: A \rightarrow C$ is also essentially smooth.

Proof. (1) Choose a finitely generated $A$-subalgebra $B^{\mathrm{f}} \subset B$ such that $B$ is a localization of $B^{\mathrm{f}}$. We can identify $U:=\operatorname{Spec} B$ with a subset of $U^{\mathrm{f}}:=\operatorname{Spec} B^{\mathrm{f}}$. Take a point $x \in U$, and let $y:=f(x) \in \operatorname{Spec} A$. Then the local $\operatorname{ring} \mathcal{O}_{U^{\mathrm{f}}, x}=$ $\mathcal{O}_{U, x}=B_{x}$ is a formally smooth $A_{y}$-algebra. According to [EGA, Chapitre IV Théorème 17.5.1] there is an open neighborhood $W$ of $x$ in $U^{\mathrm{f}}$ which is smooth over Spec $A$. Choose an element $b \in B^{\mathrm{f}}$ such that the localization $B^{\mathrm{f}}\left[b^{-1}\right]$ satisfies $x \in \operatorname{Spec} B^{\mathrm{f}}\left[b^{-1}\right] \subset W$. Then $B^{\mathrm{f}}\left[b^{-1}\right]$ is a smooth $A$-algebra, $B\left[b^{-1}\right]$ is a localization of $B^{\mathrm{f}}\left[b^{-1}\right]$, Spec $B\left[b^{-1}\right]$ is open in $\operatorname{Spec} B$, and $x \in \operatorname{Spec} B\left[b^{-1}\right]$. Finally let $i$ be an index corresponding to the point $x$, and define $B_{i}^{\mathrm{sm}}:=B^{\mathrm{f}}\left[b^{-1}\right]$ and $B_{i}:=B\left[b^{-1}\right]$.

(2) This follows from (1).

(3) See [EGA, Chapitre 0IV Proposition 20.7.4].

(4) Both conditions in Definition 3.1 are transitive.

Definition 3.3. Let $f^{*}: A \rightarrow B$ be an essentially smooth homomorphism. If $\operatorname{rank}_{B} \Omega_{B / A}^{1}=n$, then $f^{*}$ is called an essentially smooth homomorphism of relative dimension $n$, and $B$ is called an essentially smooth A-algebra of relative dimension $n$.

By Proposition 3.2(3), an essentially étale homomorphism is the same as an essentially smooth homomorphism of relative dimension 0 .

Proposition 3.4. Suppose $f^{*}: A \rightarrow B$ and $g^{*}: B \rightarrow C$ are essentially smooth homomorphisms of relative dimensions $m$ and $n$, respectively. Then $g^{*} \circ f^{*}: A \rightarrow C$ is an essentially smooth homomorphism of relative dimension $m+n$, and there is a canonical isomorphism of $C$-modules $\Omega_{C / A}^{m+n} \cong \Omega_{B / A}^{m} \otimes_{B} \Omega_{C / B}^{n}$.

Proof. By [EGA, Chapitre 0 $0_{\text {IV }}$ Théorème 20.5.7] the sequence of $C$-modules

$$
0 \rightarrow C \otimes_{B} \Omega_{B / A}^{1} \rightarrow \Omega_{C / A}^{1} \rightarrow \Omega_{C / B}^{1} \rightarrow 0
$$

is split-exact. Choose any splitting $s: \Omega_{C / B}^{1} \rightarrow \Omega_{C / A}^{1}$. Then the homomorphism

$$
\begin{gathered}
\Omega_{B / A}^{m} \otimes_{B} \Omega_{C / B}^{n} \rightarrow \Omega_{C / A}^{m+n}, \\
\left(\beta_{1} \wedge \cdots \wedge \beta_{m}\right) \otimes\left(\gamma_{1} \wedge \cdots \wedge \gamma_{n}\right) \mapsto \beta_{1} \wedge \cdots \wedge \beta_{m} \wedge s\left(\gamma_{1}\right) \wedge \cdots \wedge s\left(\gamma_{n}\right)
\end{gathered}
$$

is bijective and independent of the lifting $s$.

Let us pause to review some facts about Koszul complexes. Suppose $\boldsymbol{a}=$ $\left(a_{1}, \ldots, a_{n}\right)$ is a sequence of elements in a ring $A$. There is an associated Koszul complex $\mathbf{K}(A, \boldsymbol{a})$, which is a super-commutative nonpositive DG algebra. As a graded algebra $\mathbf{K}(A, \boldsymbol{a})$ is the exterior algebra over the ring $A$ of the free module $\bigoplus_{i=1}^{n} A \tilde{a}_{i}$, where $\tilde{a}_{1}, \ldots, \tilde{a}_{n}$ are variables of degree -1 . (In the language of Section 1 we can say that $\mathbf{K}(A, \boldsymbol{a})=A\left[\tilde{a}_{1}, \ldots, \tilde{a}_{n}\right]$, the free super-commutative algebra on these odd generators.) The differential is $\mathrm{d}\left(\tilde{a}_{i}\right):=a_{i} \in \mathbf{K}^{0}(A, \boldsymbol{a})=A$. 
Suppose $\boldsymbol{a}$ is a regular sequence in $A$. Let $J$ be the ideal generated by $\boldsymbol{a}$, and let $B:=A / J$, the quotient ring. Then the augmentation $\mathbf{K}(A, \boldsymbol{a}) \rightarrow B$ is a quasiisomorphism, and so $\mathbf{K}(A, \boldsymbol{a})$ is a free resolution of $B$ as an $A$-module. It follows that for any $A$-module $M$ we have

$$
\operatorname{Ext}_{A}^{p}(B, M)=\mathrm{H}^{p} \operatorname{Hom}_{A}(\mathbf{K}(A, \boldsymbol{a}), M) .
$$

Given an element $\mu \in M$ we define the generalized fraction

$$
\left[\begin{array}{l}
\mu \\
\boldsymbol{a}
\end{array}\right] \in \mathrm{H}^{n} \operatorname{Hom}_{A}(\mathbf{K}(A, \boldsymbol{a}), M)=\operatorname{Ext}_{A}^{n}(B, M)
$$

to be the cohomology class of the homomorphism $\mathbf{K}^{-n}(A, \boldsymbol{a}) \rightarrow M, \tilde{a}_{1} \wedge \cdots \wedge \tilde{a}_{n} \mapsto \mu$.

The $B$-module $J / J^{2}$ is free, with basis the images $\bar{a}_{1}, \ldots, \bar{a}_{n}$ of the regular sequence. Let us write $\operatorname{det}(\overline{\boldsymbol{a}}):=\bar{a}_{1} \wedge \cdots \wedge \bar{a}_{n} \in \bigwedge_{B}^{n}\left(J / J^{2}\right)$. Define the $B$-module

$$
\boldsymbol{\omega}_{B / A}:=\operatorname{Hom}_{B}\left(\bigwedge_{B}^{n}\left(J / J^{2}\right), B\right),
$$

which is free of rank 1 with basis $\frac{1}{\operatorname{det}(\overline{\boldsymbol{a}})}$. Then according to [RD, Proposition III.7.2] the map

$$
\operatorname{Ext}_{A}^{n}(B, M) \rightarrow \boldsymbol{\omega}_{B / A} \otimes_{A} M, \quad\left[\begin{array}{l}
\mu \\
\boldsymbol{a}
\end{array}\right] \mapsto \frac{1}{\operatorname{det}(\overline{\boldsymbol{a}})} \otimes \mu,
$$

is an isomorphism, and moreover it is independent of the regular sequence. Let us recall the argument: if $\boldsymbol{a}^{\prime}$ is another regular sequence generating $J$, then there is an invertible matrix $\boldsymbol{g}=\left[g_{i, j}\right]$ with entries in $A$ relating the two sequences, i.e. $a_{i}^{\prime}=\sum_{j} g_{i, j} a_{j}$. Then $\left[\begin{array}{c}\mu \\ \boldsymbol{a}^{\prime}\end{array}\right]=\operatorname{det}(\boldsymbol{g})^{-1}\left[\begin{array}{l}\mu \\ \boldsymbol{a}\end{array}\right]$, and likewise $\frac{1}{\operatorname{det}\left(\overline{\boldsymbol{a}}^{\prime}\right)}=\operatorname{det}(\boldsymbol{g})^{-1} \frac{1}{\operatorname{det}(\overline{\boldsymbol{a}})}$. Therefore the map is the same.

Clearly $\operatorname{Ext}_{A}^{p}(B, M)=0$ for all $p>n$. Furthermore, it is shown in [RD, Proposition III.7.2] that if $M$ is a flat $A$-module, then $\operatorname{Ext}_{A}^{p}(B, M)=0$ for all $p<n$.

From here until Theorem 3.14 (inclusive) we consider a flat ring homomorphism $A \rightarrow B$, and we let $B^{\mathrm{e}}:=B \otimes_{A} B$. The diagonal embedding is $\Delta: \operatorname{Spec} B \hookrightarrow$ $\operatorname{Spec} B^{\mathrm{e}}$, and we define $J:=\operatorname{Ker}\left(\Delta^{*}: B^{\mathrm{e}} \rightarrow B\right)$. Given an element $s \in B^{\mathrm{e}}$ we write $B\left[s^{-1}\right]:=B\left[\Delta^{*}(s)^{-1}\right]$ and

$$
J\left[s^{-1}\right]:=\operatorname{Ker}\left(B^{\mathrm{e}}\left[s^{-1}\right] \rightarrow B\left[s^{-1}\right]\right) \cong B^{\mathrm{e}}\left[s^{-1}\right] \otimes_{B^{\mathrm{e}}} J .
$$

Lemma 3.6. Assume $A \rightarrow B$ is essentially smooth of relative dimension $n$. Then $\Delta$ is a regular closed immersion. Namely there exist finitely many elements $s_{i} \in B^{\mathrm{e}}$ such that:

(i) $\operatorname{Spec} B=\bigcup_{i} \operatorname{Spec} B\left[s_{i}^{-1}\right]$.

(ii) For any $i$ the ideal $J\left[s_{i}^{-1}\right]$ is generated by a regular sequence of length $n$ in $B^{\mathrm{e}}\left[s_{i}^{-1}\right]$.

Proof. Choose any $x \in \operatorname{Spec} B$. By Proposition 3.2(1) there are rings $B^{\prime}$ and $B^{\text {sm }}$ such that $A \rightarrow B^{\mathrm{sm}}$ is smooth of relative dimension $n ; B^{\mathrm{sm}} \rightarrow B^{\prime}$ is a localization; Spec $B^{\prime} \subset \operatorname{Spec} B$ is open; and $x \in \operatorname{Spec} B^{\prime}$. We may assume that $B^{\prime}=B\left[r^{-1}\right]$ for some $r \in B$.

Since $A \rightarrow B^{\mathrm{sm}}$ is smooth of relative dimension $n$, according to [EGA, Chapitre IV Proposition 17.12.4] there exists an element $t \in B^{\mathrm{sm}} \otimes_{A} B^{\mathrm{sm}}$ such that $x \in$ Spec $B^{\mathrm{sm}}\left[t^{-1}\right]$, and the ideal

$$
J^{\mathrm{sm}}\left[t^{-1}\right]:=\operatorname{Ker}\left(\left(B^{\mathrm{sm}} \otimes_{A} B^{\mathrm{sm}}\right)\left[t^{-1}\right] \rightarrow B^{\mathrm{sm}}\left[t^{-1}\right]\right)
$$

is generated by a regular sequence of length $n$. 
Now there is a ring homomorphism

$$
B^{\mathrm{sm}} \otimes_{A} B^{\mathrm{sm}} \rightarrow B\left[r^{-1}\right] \otimes_{A} B\left[r^{-1}\right]=B^{\mathrm{e}}\left[(r \otimes r)^{-1}\right] .
$$

Using it we can write $t=t_{0}(r \otimes r)^{-l} \in B^{\mathrm{e}}\left[(r \otimes r)^{-1}\right]$ for some nonnegative integer $l$ and an element $t_{0} \in B^{\mathrm{e}}$. Define $s_{x}:=t_{0}(r \otimes r) \in B^{\mathrm{e}}$. Then the homomorphism $B^{\mathrm{sm}} \otimes_{A} B^{\mathrm{sm}} \rightarrow B^{\mathrm{e}}\left[s_{x}^{-1}\right]$ is flat, and the ideal

$$
J\left[s_{x}^{-1}\right] \cong B^{\mathrm{e}}\left[s_{x}^{-1}\right] \otimes_{B^{\mathrm{sm}} \otimes_{A} B^{\mathrm{sm}}} J^{\mathrm{sm}}\left[t^{-1}\right]
$$

is generated by the same regular sequence of length $n$.

Going over all $x \in \operatorname{Spec} B$ we thus obtain a set $\left\{s_{x}\right\}$ of elements of $B^{\mathrm{e}}$ satisfying the two conditions (i), (ii). Since $\operatorname{Spec} B$ is quasi-compact we can select a finite subset.

Remark 3.7. In general the ring $B^{\mathrm{e}}\left[s^{-1}\right]$ is not the same as the $\operatorname{ring} B\left[s^{-1}\right] \otimes_{A}$ $B\left[s^{-1}\right]$. For instance, take $A:=\mathbb{R}$ and $B:=\mathbb{C}$ (so $n=0$ here). Let $s:=$ $\mathbf{i} \otimes 1+1 \otimes \mathbf{i} \in B^{\mathrm{e}}$. Then $B^{\mathrm{e}}\left[s^{-1}\right]=B\left[s^{-1}\right]=B$, but $B\left[s^{-1}\right] \otimes_{A} B\left[s^{-1}\right]=B^{\mathrm{e}} \nsucceq B$.

Take some $s \in B^{\mathrm{e}}$, and let $\boldsymbol{b}=\left(b_{1}, \ldots, b_{n}\right)$ be a sequence of elements in $B^{\mathrm{e}}\left[s^{-1}\right]$. We define

$$
\mathrm{d}(\boldsymbol{b}):=\mathrm{d}\left(b_{1}\right) \wedge \cdots \wedge \mathrm{d}\left(b_{n}\right) \in \Omega_{B^{\mathrm{e}}\left[s^{-1}\right] / A}^{n} .
$$

The de Rham complex $\Omega_{B / A}$ is a DG $A$-algebra (which lives in nonnegative degrees!). The $A$-algebra homomorphism $\mathrm{p}_{2}^{*}: B \rightarrow B^{\mathrm{e}}, b \mapsto 1 \otimes b$, extends to a DG algebra homomorphism $\mathrm{p}_{2}^{*}: \Omega_{B / A} \rightarrow \Omega_{B^{\mathrm{e}} / A}$. Thus given an element $\beta \in \Omega_{B / A}^{n}$ we obtain an element $\mathrm{p}_{2}^{*}(\beta) \in \Omega_{B^{\mathrm{e}} / A}^{n}$. Clearly $\left(\Delta^{*} \circ \mathrm{p}_{2}^{*}\right)(\beta)=\beta$.

Lemma 3.8. Assume $A \rightarrow B$ is essentially smooth of relative dimension $n$, and $s \in B^{\mathrm{e}}$ is such that the ideal $J\left[s^{-1}\right]$ is generated by a regular sequence.

(1) There is a unique $B\left[s^{-1}\right]$-linear isomorphism

$$
\Omega_{B\left[s^{-1}\right] / A}^{n} \stackrel{\simeq}{\longrightarrow} \operatorname{Ext}_{B^{\mathrm{e}}\left[s^{-1}\right]}^{n}\left(B\left[s^{-1}\right], \Omega_{B^{\mathrm{e}}\left[s^{-1}\right] / A}^{2 n}\right)
$$

such that

$$
\beta \mapsto\left[\begin{array}{c}
\mathrm{d}(\boldsymbol{b}) \wedge \mathrm{p}_{2}^{*}(\beta) \\
\boldsymbol{b}
\end{array}\right]
$$

for any regular sequence $\boldsymbol{b}=\left(b_{1}, \ldots, b_{n}\right)$ generating $J\left[s^{-1}\right]$ and any $\beta \in$ $\Omega_{B / A}^{n}$.

(2) Let $M$ be any $B^{\mathrm{e}}\left[s^{-1}\right]$-module. Then

$$
\operatorname{Ext}_{B^{\mathrm{e}}\left[s^{-1}\right]}^{p}\left(B\left[s^{-1}\right], M\right)=0
$$

for all $p>n$. Moreover, if $M$ is flat, then this vanishing also holds for $p<n$.

Proof. (1) First we observe that there's an isomorphism of $B$-modules

$$
\Omega_{B / A}^{1} \stackrel{\simeq}{\longrightarrow} J / J^{2}, \quad \mathrm{~d}(b) \mapsto b \otimes 1-1 \otimes b \bmod J^{2}
$$

for $b \in B$. In this way we get an isomorphism $\operatorname{Hom}_{B}\left(\Omega_{B / A}^{n}, B\right) \cong \boldsymbol{\omega}_{B / B^{\mathrm{e}}}$. The derivation d : $B^{\mathrm{e}} \rightarrow \Omega_{B^{\mathrm{e}} / A}^{1}$ restricts to a function $\mathrm{d}: J \rightarrow \Omega_{B^{\mathrm{e}} / A}^{1}$ such that $\mathrm{d}\left(J^{2}\right) \subset J \cdot \Omega_{B^{\mathrm{e}} / A}^{1}$, and this gives rise to a split exact sequence of $B$-modules

$$
0 \rightarrow J / J^{2} \stackrel{\overline{\mathrm{d}}}{\rightarrow} B \otimes_{B^{\mathrm{e}}} \Omega_{B^{\mathrm{e}} / A}^{1} \rightarrow \Omega_{B / A}^{1} \rightarrow 0 .
$$


In this setup an element $b \in J \subset B^{\mathrm{e}}$ will have images $\bar{b} \in J / J^{2}$ and $\mathrm{d}(b) \in$ $\Omega_{B^{\mathrm{e}} / A}^{1}$, and these are related by the formula $\overline{\mathrm{d}}(\bar{b})=1 \otimes \mathrm{d}(b) \in B \otimes_{B^{\mathrm{e}}} \Omega_{B^{\mathrm{e}} / A^{1}}^{1}$. Taking determinants (i.e. top degree exterior powers) in (3.9) and (3.10) we obtain a canonical isomorphism

$$
B \otimes_{B^{\mathrm{e}}} \Omega_{B^{\mathrm{e}} / A}^{2 n} \cong \Omega_{B / A}^{n} \otimes_{B} \Omega_{B / A}^{n} .
$$

For any sequence $\boldsymbol{b}=\left(b_{1}, \ldots, b_{n}\right)$ of elements in $J$ and differential form $\beta \in \Omega_{B / A}^{n}$ this isomorphism sends $1 \otimes\left(\mathrm{d}(\boldsymbol{b}) \wedge \mathrm{p}_{2}^{*}(\beta)\right) \mapsto \operatorname{det}(\overline{\boldsymbol{b}}) \otimes \beta$.

Now consider the isomorphisms (3.5) and (3.11), but with updated entries. Here we look at the surjective ring homomorphism $B^{\mathrm{e}}\left[s^{-1}\right] \rightarrow B\left[s^{-1}\right]$, and the $B^{\mathrm{e}}\left[s^{-1}\right]$ module $\Omega_{B \mathrm{e}\left[s^{-1}\right] / A}^{2 n}$. We get

$$
\begin{aligned}
& \operatorname{Ext}_{B^{\mathrm{e}}\left[s^{-1}\right]}^{n}\left(B\left[s^{-1}\right], \Omega_{B^{\mathrm{e}}\left[s^{-1}\right] / A}^{2 n}\right) \\
& \quad \cong \boldsymbol{\omega}_{B\left[s^{-1}\right] / B^{\mathrm{e}}\left[s^{-1}\right]} \otimes_{B\left[s^{-1}\right]} \Omega_{B\left[s^{-1}\right] / A}^{n} \otimes_{B\left[s^{-1}\right]} \Omega_{B\left[s^{-1}\right] / A}^{n} .
\end{aligned}
$$

Suppose the sequence $\boldsymbol{b}=\left(b_{1}, \ldots, b_{n}\right)$ is regular and generates $J\left[s^{-1}\right]$. Then the element $\operatorname{det}(\overline{\boldsymbol{b}})$ generates the $B\left[s^{-1}\right]$-module

$$
\bigwedge_{B\left[s^{-1}\right]}^{n}\left(J\left[s^{-1}\right] / J\left[s^{-1}\right]^{2}\right) \cong \Omega_{B\left[s^{-1}\right] / A}^{n}
$$

Also any element of $B\left[s^{-1}\right] \otimes_{B^{\mathrm{e}}\left[s^{-1}\right]} \Omega_{B^{\mathrm{e}}\left[s^{-1}\right] / A}^{2}$ can be expressed as $\mathrm{d}(\boldsymbol{b}) \wedge \mathrm{p}_{2}^{*}\left(s^{-l} \beta\right)$ for some $\beta \in \Omega_{B / A}^{n}$ and $l \geq 0$. The isomorphism (3.12) sends

$$
\left[\begin{array}{c}
\mathrm{d}(\boldsymbol{b}) \wedge \mathrm{p}_{2}^{*}\left(s^{-l} \beta\right) \\
\boldsymbol{b}
\end{array}\right] \mapsto \frac{1}{\operatorname{det}(\overline{\boldsymbol{b}})} \otimes \operatorname{det}(\overline{\boldsymbol{b}}) \otimes s^{-l} \beta .
$$

On the other hand the map

$$
s^{-l} \beta \mapsto \frac{1}{\operatorname{det}(\overline{\boldsymbol{b}})} \otimes \operatorname{det}(\overline{\boldsymbol{b}}) \otimes s^{-l} \beta
$$

is a $B\left[s^{-1}\right]$-linear isomorphism

$$
\Omega_{B\left[s^{-1}\right] / A}^{n} \stackrel{\simeq}{\longrightarrow} \boldsymbol{\omega}_{B\left[s^{-1}\right] / B^{\mathrm{e}}\left[s^{-1}\right]} \otimes_{B\left[s^{-1}\right]} \Omega_{B\left[s^{-1}\right] / A}^{n} \otimes_{B\left[s^{-1}\right]} \Omega_{B\left[s^{-1}\right] / A}^{n}
$$

which is evidently independent of the regular sequence $\boldsymbol{b}$. The isomorphism we want is the composition of (3.12) and (3.13).

(2) Take $s$ and $\boldsymbol{b}$ as in part (1). We can use the Koszul complex $\mathbf{K}\left(B^{\mathrm{e}}\left[s^{-1}\right], \boldsymbol{b}\right)$ to calculate $\operatorname{Ext}_{B^{\mathrm{e}}\left[s^{-1}\right]}^{p}\left(B\left[s^{-1}\right], M\right)$. The assertions now follow from the general facts about Koszul complexes mentioned earlier; cf. [RD, Proposition III.7.2].

Theorem 3.14. Let $A \rightarrow B$ be an essentially smooth homomorphism of relative dimension $n$. Define $B^{\mathrm{e}}:=B \otimes_{A} B$.

(1) The $B^{\mathrm{e}}$-module $B$ has finite projective dimension.

(2) One has $\operatorname{Ext}_{B^{\mathrm{e}}}^{p}\left(B, \Omega_{B^{\mathrm{e}} / A}^{2 n}\right)=0$ for all $p \neq n$.

(3) There is a unique B-linear isomorphism

$$
\Omega_{B / A}^{n} \stackrel{\simeq}{\longrightarrow} \operatorname{Ext}_{B^{\mathrm{e}}}^{n}\left(B, \Omega_{B^{\mathrm{e}} / A}^{2 n}\right)
$$

which coincides with the isomorphism of Lemma 3.8(1) after inverting any element $s \in B^{\mathrm{e}}$ as described there. 
Proof. (1), (2) We shall prove that for any $B^{\mathrm{e}}$-module $M$ and any $p>n$ the module $\operatorname{Ext}_{B^{\mathrm{e}}}^{p}(B, M)$ vanishes, and if $M$ is flat there is also vanishing for $p<n$. Let $\operatorname{Spec} B=\bigcup_{i}$ Spec $B\left[s_{i}^{-1}\right]$ be the open covering from the Lemma 3.6. It suffices to show that $B\left[s_{i}^{-1}\right] \otimes_{B} \operatorname{Ext}_{B^{\mathrm{e}}}^{p}(B, M)=0$ for all $i$ and all $p$ in the corresponding range of integers. But this was done in Lemma 3.8(2).

(3) Uniqueness is clear, in view of Lemma 3.6. Regarding existence: due to the independence on regular sequences, the isomorphisms of Lemma 3.8(1) on the open sets Spec $B\left[s_{i}^{-1}\right]$ can be glued.

Finally a result about essentially étale homomorphisms.

Proposition 3.15. Let $A \rightarrow B$ be an essentially étale homomorphism, and define $B^{\mathrm{e}}:=B \otimes_{A} B$. Let $\mu: B^{\mathrm{e}} \rightarrow B$ be the multiplication map, and let $I:=\operatorname{Ker}(\mu)$. We view $\operatorname{Hom}_{B^{\mathrm{e}}}\left(B, B^{\mathrm{e}}\right)$ as an ideal of $B^{\mathrm{e}}$, i.e. the annihilator of $I$. Then there is a unique ring isomorphism $\nu: B \times B^{\prime} \stackrel{\simeq}{\longrightarrow} B^{\mathrm{e}}$ such that $\nu\left(B^{\prime}\right)=I, \nu(B)=$ $\operatorname{Hom}_{B^{\mathrm{e}}}\left(B, B^{\mathrm{e}}\right)$, and $\mu \circ \nu: B \rightarrow B$ is the identity.

Proof. Uniqueness: once we know that $B^{\mathrm{e}}=I \oplus \operatorname{Hom}_{B^{\mathrm{e}}}\left(B, B^{\mathrm{e}}\right)$ as $B^{\mathrm{e}}$-modules, the ring $B^{\prime}$ and the isomorphism $\nu$ are determined.

We need to construct $\nu$. First let's assume that $A \rightarrow B$ is étale (i.e. $B$ is a finitely generated $A$-algebra). Let $f^{*}: B \rightarrow B^{\mathrm{e}}$ be the homomorphism $f^{*}(b):=b \otimes 1$. Define $X:=\operatorname{Spec} B^{\mathrm{e}}$ and $Y:=\operatorname{Spec} B$; so $f: X \rightarrow Y$ is étale and separated. The diagonal morphism $\Delta: Y \rightarrow X$ is a section of $f$, and $\Delta^{*}=\mu$. According to [EGA, Corollaire IV.17.9.3] the morphism $\Delta$ is a closed and open immersion, so that $X=\Delta(Y) \coprod Z$ for some affine scheme $Z=\operatorname{Spec} B^{\prime}$. We get a ring isomorphism $\nu: B \times B^{\prime} \stackrel{\simeq}{\longrightarrow} B^{\text {e }}$ such that $\nu \circ \mu: B \rightarrow B$ is the identity. Let $\epsilon, \epsilon^{\prime} \in B^{\mathrm{e}}$ be the corresponding orthogonal idempotent elements, so that $\nu(B)=B^{\mathrm{e}} \cdot \epsilon$ and $\nu\left(B^{\prime}\right)=B^{\mathrm{e}} \cdot \epsilon^{\prime}$. Then $B^{\mathrm{e}} \cdot \epsilon^{\prime}=I$, and the annihilator of $I$ in $B^{\mathrm{e}}$ is precisely $B^{\mathrm{e}} \cdot \epsilon$.

Next lets look at a localization $B \rightarrow C$. Define $C^{\mathrm{e}}:=C \otimes_{A} C, \mu_{C}: C^{\mathrm{e}} \rightarrow C$ the multiplication map, $I_{C}:=\operatorname{Ker}\left(\mu_{C}\right)$ and $C^{\prime}:=C^{\mathrm{e}} \otimes_{B^{\mathrm{e}}} B^{\prime}$. There is a factorization $\nu_{C}: C \times C^{\prime} \stackrel{\simeq}{\longrightarrow} C^{\mathrm{e}}$ obtained from $\nu$ by the tensor operation $C^{\mathrm{e}} \otimes_{B^{\mathrm{e}}}-$. Note that $I_{C}=C^{\mathrm{e}} \cdot I$, and so this decomposition can be characterized as $\nu_{C}\left(C^{\prime}\right)=I_{C}, \nu_{C}(C)$ is the annihilator of $I_{C}$, and $\mu_{C} \circ \nu_{C}: C \rightarrow C$ is the identity.

Now for the general case. According to Proposition 3.2(1) we have an open covering $\operatorname{Spec} B=\bigcup_{i}$ Spec $B_{i}$, such that for every $i$ the homomorphism $A \rightarrow B_{i}$ is the composition of an étale homomorphism $A \rightarrow B_{i}^{\mathrm{sm}}$ and a localization $B_{i}^{\mathrm{sm}} \rightarrow B_{i}$. As explained in the previous paragraph there are factorizations $\nu_{i}: B_{i} \times B_{i}^{\prime} \stackrel{\simeq}{\longrightarrow}$ $B_{i} \otimes_{A} B_{i}$, and these are compatible with further localization. Therefore they can be glued into a global factorization $\nu: B \times B^{\prime} \stackrel{\simeq}{\longrightarrow} B \otimes_{A} B$.

\section{Rigid COMPLEXES}

In this section we introduce the main concept of the paper, namely rigid complexes. This concept is due to Van den Bergh [VdB].

First a comment on bounded complexes. Let $B$ be a ring and $M \in \mathrm{D}(\operatorname{Mod} B)$. If $M$ has bounded cohomology, then, after replacing it with the isomorphic complex $\tau^{\geq i} \tau^{\leq j} M$ for $i \gg 0$ and $j \ll 0$, we can assume that $M \in \mathrm{D}^{\mathrm{b}}(\operatorname{Mod} B)$. Likewise for $\mathrm{D}^{+}(\operatorname{Mod} B)$. Such considerations will be made implicitly throughout the paper.

For a noetherian ring $A$ we denote by $\mathrm{D}_{\mathrm{f}}^{\mathrm{b}}(\operatorname{Mod} A)$ the derived category of bounded complexes with finitely generated cohomologies. 
Let $A$ be a ring and let $B$ be an $A$-algebra. In Section 2 we constructed a functor $\mathrm{Sq}_{B / A}: \mathrm{D}(\operatorname{Mod} B) \rightarrow \mathrm{D}(\operatorname{Mod} B)$, the squaring operation (see Definition 2.6). When $A$ is a field one has the easy formula

$$
\mathrm{Sq}_{B / A} M=\mathrm{RHom}_{B \otimes_{A} B}\left(B, M \otimes_{A} M\right)
$$

(see Corollary 2.8). The squaring is functorial for algebra homomorphisms too. Given a homomorphism of algebras $f^{*}: B \rightarrow C$, complexes $M \in \mathrm{D}(\operatorname{Mod} B)$ and $N \in \mathrm{D}(\operatorname{Mod} C)$, and a morphism $\phi: N \rightarrow M$ in $\mathrm{D}(\operatorname{Mod} B)$, there is an induced morphism $\mathrm{Sq}_{f^{*} / A}(\phi): \mathrm{Sq}_{C / A} N \rightarrow \mathrm{Sq}_{B / A} M$ in $\mathrm{D}(\operatorname{Mod} B)$. Again when $A$ is a field the formula for $\mathrm{Sq}_{f * / A}$ is obvious; complications arise only when the base $\operatorname{ring} A$ is not a field.

Definition 4.1. Let $A$ be a ring, let $B$ be a noetherian $A$-algebra, and let $M \in$ $\mathrm{D}(\operatorname{Mod} B)$.

(1) A rigidifying isomorphism for $M$ relative to $A$ is an isomorphism

$$
\rho: M \stackrel{\simeq}{\longrightarrow} \mathrm{Sq}_{B / A} M
$$

in $\mathrm{D}(\operatorname{Mod} B)$.

(2) If $M \in \mathrm{D}_{\mathrm{f}}^{\mathrm{b}}(\operatorname{Mod} B)$ and it has finite flat dimension over $A$, then the pair $(M, \rho)$ in part (1) is called a rigid complex over $B$ relative to $A$.

Example 4.2. Take $B=M:=A$. Since $\mathrm{Sq}_{A / A} A=A$ it follows that $A$ has a tautological rigidifying isomorphism $\rho_{A}^{\mathrm{tau}}: A \stackrel{\simeq}{\longrightarrow} \mathrm{Sq}_{A / A} A$. We call $\left(A, \rho_{A}^{\mathrm{tau}}\right)$ the tautological rigid complex over $A$ relative to $A$.

Definition 4.3. Let $A$ be a ring, let $B, C$ be noetherian $A$-algebras, let $f^{*}: B \rightarrow C$ be a homomorphism of $A$-algebras, and let $\left(M, \rho_{M}\right)$ and $\left(N, \rho_{N}\right)$ be rigid complexes over $B$ and $C$, respectively, both relative to $A$. A morphism $\phi: N \rightarrow M$ in $\mathrm{D}(\operatorname{Mod} B)$ is called a rigid trace morphism relative to $A$ if the diagram

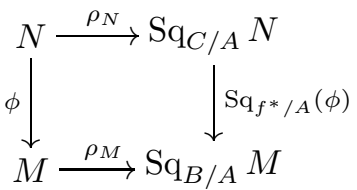

of morphisms in $\mathrm{D}(\operatorname{Mod} B)$ is commutative. If $B=C$ (and $f^{*}$ is the identity), then we say $\phi: N \rightarrow M$ is a rigid morphism over $B$ relative to $A$.

It is easy to see that the composition of two rigid trace morphisms relative to $A$ is again a rigid trace morphism relative to $A$. In particular, for a fixed $A$-algebra $B$ the rigid complexes over $B$ relative to $A$ form a category, which we denote by $\mathrm{D}_{\mathrm{f}}^{\mathrm{b}}(\operatorname{Mod} B)_{\mathrm{rig} / A}$.

The importance of rigid complexes is captured by the next result.

Theorem 4.4. Let $A$ be a ring, let $B$ be a noetherian $A$-algebra, and let $(M, \rho) \in$ $\mathrm{D}_{\mathrm{f}}^{\mathrm{b}}(\operatorname{Mod} B)_{\mathrm{rig} / A}$. Assume the canonical homomorphism $B \rightarrow \operatorname{Hom}_{\mathrm{D}(\operatorname{Mod} B)}(M, M)$ is bijective. Then the only automorphism of $(M, \rho)$ in $\mathrm{D}_{\mathrm{f}}^{\mathrm{b}}(\operatorname{Mod} B)_{\mathrm{rig} / A}$ is the identity $\mathbf{1}_{M}$.

Proof. Let $\phi: M \rightarrow M$ be a rigid isomorphism. So $\phi=b \mathbf{1}_{M}$ for an invertible element $b \in B$. Then

$$
\mathrm{Sq}_{B / A}(\phi) \circ \rho=\rho \circ \phi=\rho \circ\left(b \mathbf{1}_{M}\right)=b \rho
$$


in $\operatorname{Hom}_{\mathrm{D}(\operatorname{Mod} B)}\left(M, \mathrm{Sq}_{B / A} M\right)$. On the other hand, using Corollary 2.7 we have

$$
\mathrm{Sq}_{B / A}(\phi) \circ \rho=\operatorname{Sq}_{B / A}\left(b \mathbf{1}_{M}\right) \circ \rho=b^{2} \operatorname{Sq}_{B / A}\left(\mathbf{1}_{M}\right) \circ \rho=b^{2} \rho \circ \mathbf{1}_{M}=b^{2} \rho .
$$

Hence $b=1$.

Remark 4.5. In our next paper [YZ4] we prove the following result, which adds to the interest in rigid complexes. Suppose $A$ is a regular finite dimensional noetherian ring. Let $B$ be an essentially finite type $A$-algebra, and assume $\operatorname{Spec} B$ is connected and nonempty. Then, up to isomorphism, the category $\mathrm{D}_{\mathrm{f}}^{\mathrm{b}}(\operatorname{Mod} B)_{\mathrm{rig} / A}$ contains exactly two objects: the zero complex, and a nonzero rigid complex $(R, \rho)$. The rigid complex $(R, \rho)$ satisfies the condition of Theorem 4.4, so it has only one automorphism. Moreover the complex $R$ is dualizing.

Let $A$ be a ring, let $B \rightarrow C$ be a homomorphism of $A$-algebras, let $M \in \mathrm{D}(\operatorname{Mod} B)$ and let $N \in \mathrm{D}(\operatorname{Mod} C)$. Choose a K-flat $\mathrm{DG}$ algebra resolution $A \rightarrow \tilde{B} \rightarrow B$ of $A \rightarrow B$, and then a K-flat DG algebra resolution $\tilde{B} \rightarrow \tilde{C} \rightarrow C$ of $\tilde{B} \rightarrow C$. This can be done by Proposition 1.7. (If $A \rightarrow B$ and $B \rightarrow C$ are flat one may just take $\tilde{B}=B$ and $\tilde{C}=C$.) Then there is a sequence of morphisms in $\mathrm{D}(\operatorname{Mod} C)$ :

$$
\begin{aligned}
& \left(\mathrm{Sq}_{B / A} M\right) \otimes_{B}^{\mathrm{L}}\left(\mathrm{Sq}_{C / B} N\right) \\
& \quad=\operatorname{RHom}_{\tilde{B} \otimes_{A} \tilde{B}}\left(B, M \otimes_{A}^{\mathrm{L}} M\right) \otimes_{\tilde{B}}^{\mathrm{L}} \operatorname{RHom}_{\tilde{C} \otimes_{\tilde{B}} \tilde{C}}\left(C, N \otimes_{\tilde{B}}^{\mathrm{L}} N\right) \\
& \quad \rightarrow^{\triangleright} \operatorname{RHom}_{\tilde{C} \otimes_{\tilde{B}} \tilde{C}}\left(C, N \otimes_{\tilde{B}}^{\mathrm{L}} N \otimes_{\tilde{B}}^{\mathrm{L}} \operatorname{RHom}_{\tilde{B} \otimes_{A} \tilde{B}}\left(B, M \otimes_{A}^{\mathrm{L}} M\right)\right) \\
& \quad \rightarrow \operatorname{RHom}_{\tilde{C} \otimes_{\tilde{B}} \tilde{C}}\left(C, \operatorname{RHom}_{\tilde{B} \otimes_{A} \tilde{B}}\left(B,\left(M \otimes_{\tilde{B}}^{\mathrm{L}} N\right) \otimes_{A}^{\mathrm{L}}\left(M \otimes_{\tilde{B}}^{\mathrm{L}} N\right)\right)\right) \\
& \quad \rightarrow^{\dagger} \operatorname{RHom}_{\tilde{C} \otimes_{\tilde{B}} \tilde{C}}\left(C, \operatorname{RHom}_{\tilde{C} \otimes_{A} \tilde{C}}\left(\tilde{C} \otimes_{\tilde{B}} \tilde{C},\left(M \otimes_{\tilde{B}}^{\mathrm{L}} N\right) \otimes_{A}^{\mathrm{L}}\left(M \otimes_{\tilde{B}}^{\mathrm{L}} N\right)\right)\right) \\
& \quad \rightarrow{ }^{\ominus} \operatorname{RHom}_{\tilde{C} \otimes_{A} \tilde{C}}\left(C,\left(M \otimes_{\tilde{B}}^{\mathrm{L}} N\right) \otimes_{A}^{\mathrm{L}}\left(M \otimes_{\tilde{B}}^{\mathrm{L}} N\right)\right) \\
& \quad=\operatorname{Sq}_{C / A}\left(M \otimes_{B}^{\mathrm{L}} N\right)
\end{aligned}
$$

defined as follows. The morphism $\diamond$ is of the form

$$
X \otimes^{\mathrm{L}} \operatorname{RHom}(Y, Z) \rightarrow \operatorname{RHom}\left(Y, X \otimes^{\mathrm{L}} Z\right) .
$$

The morphism $\nabla$ is a double application of (4.7). The morphism $\dagger$ is actually an isomorphism: it is Hom-tensor adjunction for the DG algebra homomorphism $\tilde{B} \otimes_{A} \tilde{B} \rightarrow \tilde{C} \otimes_{A} \tilde{C}$, plus the fact that

$$
\tilde{C} \otimes_{\tilde{B}} \tilde{C} \cong\left(\tilde{C} \otimes_{A} \tilde{C}\right) \otimes_{\tilde{B} \otimes_{A} \tilde{B}} B .
$$

The morphism $\varnothing$ is also an isomorphism, being a Hom-tensor adjunction for the DG algebra homomorphism $\tilde{C} \otimes_{A} \tilde{C} \rightarrow \tilde{C} \otimes_{\tilde{B}} \tilde{C}$.

Lemma 4.8. Let $A$ be a ring, let $f^{*}: B \rightarrow C$ be a homomorphism of A-algebras, let $M \in \mathrm{D}(\operatorname{Mod} B)$ and let $N \in \mathrm{D}(\operatorname{Mod} C)$. Then the morphism

$$
\smile_{f^{*} ; M, N}:\left(\mathrm{Sq}_{B / A} M\right) \otimes_{B}^{\mathrm{L}}\left(\mathrm{Sq}_{C / B} N\right) \rightarrow \mathrm{Sq}_{C / A}\left(M \otimes_{B}^{\mathrm{L}} N\right)
$$

in $\mathrm{D}(\operatorname{Mod} C)$ that was constructed in (4.6) is independent of the resolutions $A \rightarrow$ $\tilde{B} \rightarrow B$ and $\tilde{B} \rightarrow \tilde{C} \rightarrow C$.

Proof. The homotopy arguments in the proofs of Theorem 2.2 apply here.

The morphism $\smile_{f ; M, N}$ is called the cup product. 
Lemma 4.9. The cup product $\smile_{f ; M, N}$ is functorial in $M$ and $N$. Namely suppose we are given a morphism $\phi: M_{1} \rightarrow M_{2}$ in $\mathrm{D}(\operatorname{Mod} B)$ and a morphism $\psi: N_{1} \rightarrow N_{2}$ in $\mathrm{D}(\operatorname{Mod} C)$. Then the diagram

$$
\begin{aligned}
& \left(\mathrm{Sq}_{B / A} M_{1}\right) \otimes \mathrm{L}_{B}^{\mathrm{L}}\left(\mathrm{Sq}_{C / B} N_{1}\right) \stackrel{\smile_{f ; M_{1}, N_{1}}}{\longrightarrow} \mathrm{Sq}_{C / A}\left(M_{1} \otimes_{B}^{\mathrm{L}} N_{1}\right) \\
& \quad \mathrm{Sq}_{B / A}(\phi) \otimes \mathrm{Sq}_{C / B}(\psi) \\
& \left(\mathrm{Sq}_{B / A} M_{2}\right) \otimes \mathrm{Sq}_{C / A}(\phi \otimes \psi) \\
& \left.\mathrm{Sq}_{C / B} N_{2}\right) \stackrel{\mathrm{Sq}_{f ; M_{2}, N_{2}}}{\longrightarrow} \mathrm{Sq}_{C / A}\left(M_{2} \otimes_{B}^{\mathrm{L}} N_{2}\right)
\end{aligned}
$$

is commutative.

Proof. Choose a semi-free DG algebra resolution $A \rightarrow \tilde{B} \rightarrow B$ of $A \rightarrow B$, and a semi-free DG algebra resolution $\tilde{B} \rightarrow \tilde{C} \rightarrow C$ of $\tilde{B} \rightarrow C$. According to Theorem 2.3 we have representations $\operatorname{Sq}_{B / A}(\phi)=\left(\mathbf{1}_{B}, \phi \otimes \phi\right), \mathrm{Sq}_{C / B}(\psi)=\left(\mathbf{1}_{C}, \psi \otimes \psi\right)$ and

$$
\mathrm{Sq}_{C / A}(\phi \otimes \psi)=\left(\left(\mathbf{1}_{C},(\phi \otimes \psi) \otimes(\phi \otimes \psi)\right) .\right.
$$

All the morphisms in (4.6) are compatible with $\phi$ and $\psi$. Hence the diagram (4.10) is commutative.

Theorem 4.11. Let $A$ be a noetherian ring, let $B$ and $C$ be essentially finite type $A$ algebras, let $f^{*}: B \rightarrow C$ be an A-algebra homomorphism, and let $M \in \mathrm{D}_{\mathrm{f}}^{\mathrm{b}}(\operatorname{Mod} B)$ and $N \in \mathrm{D}_{\mathrm{f}}^{\mathrm{b}}(\operatorname{Mod} C)$. Assume all three conditions (i), (ii) and (iii) below hold.

(i) The complex $M$ has finite flat dimension over $A$, and $\mathrm{H}_{\mathrm{Sq}_{B} A} M$ is bounded.

(ii) The complex $N$ has finite flat dimension over $B$.

(iii) Either (a), (b) or (c) is satisfied:

(a) $B \rightarrow C$ is essentially smooth.

(b) $M$ has finite flat dimension over $B$.

(c) The the canonical morphism $B \rightarrow \operatorname{RHom}_{B}(M, M)$ is an isomorphism.

Then:

(1) The complex $M \otimes_{B}^{\mathrm{L}} N$ is in $\mathrm{D}_{\mathrm{f}}^{\mathrm{b}}(\operatorname{Mod} C)$, and it has finite flat dimension over $A$.

(2) The cup product morphism

$$
\smile_{f ; M, N}:\left(\mathrm{Sq}_{B / A} M\right) \otimes_{B}^{\mathrm{L}}\left(\mathrm{Sq}_{C / B} N\right) \rightarrow \mathrm{Sq}_{C / A}\left(M \otimes_{B}^{\mathrm{L}} N\right)
$$

is an isomorphism.

Proof. (1) The cohomology modules $\mathrm{H}^{i}\left(M \otimes_{B}^{\mathrm{L}} N\right)$ are finitely generated over $C$ because the rings are noetherian. Since $M$ has finite flat dimension over $A$ and $N$ has finite flat dimension over $B$ (cf. Definition 4.1), it follows that $M \otimes_{B}^{\mathrm{L}} N \in$ $\mathrm{D}(\operatorname{Mod} C)$ has finite flat dimension over $A$. In particular $\mathrm{H}\left(M \otimes_{B}^{\mathrm{L}} N\right)$ is bounded, and so we can assume that $M \otimes_{B}^{\mathrm{L}} N \in \mathrm{D}_{\mathrm{f}}^{\mathrm{b}}(\operatorname{Mod} C)$.

(2) As was already mentioned the morphisms $\dagger$ and $\varnothing$ in (4.6) are automatically isomorphisms.

Let us consider the morphism $\nabla$. In order to prove that the morphism

$$
\begin{aligned}
& N \otimes_{\tilde{B}}^{\mathrm{L}} N \otimes_{\tilde{B}}^{\mathrm{L}} \operatorname{RHom}_{\tilde{B} \otimes_{A} \tilde{B}}\left(B, M \otimes_{A}^{\mathrm{L}} M\right) \\
& \quad \rightarrow \operatorname{RHom}_{\tilde{B} \otimes_{A} \tilde{B}}\left(B,\left(M \otimes_{\tilde{B}}^{\mathrm{L}} N\right) \otimes_{A}^{\mathrm{L}}\left(M \otimes_{\tilde{B}}^{\mathrm{L}} N\right)\right)
\end{aligned}
$$

in $\tilde{\mathrm{D}}\left(\mathrm{DGMod} \tilde{C} \otimes_{A} \tilde{C}\right)$ is an isomorphism, we can forget the $\tilde{C} \otimes_{A} \tilde{C}$-module structure, and consider this as a morphism in $\mathrm{D}(\operatorname{Nod} A)$. According to Corollary 1.10 the 
algebra $\mathrm{H}^{0}\left(\tilde{B} \otimes_{A} \tilde{B}\right) \cong B \otimes_{A} B$ is noetherian, and each $\mathrm{H}^{i}\left(\tilde{B} \otimes_{A} \tilde{B}\right)$ is a finitely generated module over it. Since $N$ has finite flat dimension over $\tilde{B}$, and both $\mathrm{H}\left(M \otimes_{A}^{\mathrm{L}} M\right)$ and $\mathrm{H}\left(\left(M \otimes_{\tilde{B}}^{\mathrm{L}} N\right) \otimes_{A}^{\mathrm{L}} M\right)$ are bounded, we can use Proposition 1.12 twice, with its condition (iii)(b), to conclude that (4.12) is an isomorphism.

Finally let's show that $\diamond$ is an isomorphism. The DG modules $N \otimes_{B}^{\mathrm{L}} N$ and $\mathrm{Sq}_{B / A} M$ have bounded cohomologies. If $B \rightarrow C$ is essentially smooth, then $\tilde{C} \otimes_{\tilde{B}} \tilde{C} \rightarrow C \otimes_{B} C$ is a quasi-isomorphism, and moreover $C$ has finite projective dimension over $C \otimes_{B} C$. Thus under either condition (iii)(a), (iii)(b) or (iii)(c) of the theorem we may apply Proposition 1.12, with its conditions (iii)(a), (iii)(b) or (iii)(c), respectively, to deduce that $\diamond$ is an isomorphism.

The next result is an immediate consequence of the theorem.

Corollary 4.13. Let $A$ be a noetherian ring, let $B$ and $C$ be essentially finite type $A$-algebras, let $f^{*}: B \rightarrow C$ be an A-algebra homomorphism, let $\left(M, \rho_{M}\right) \in$ $\mathrm{D}_{\mathrm{f}}^{\mathrm{b}}(\operatorname{Mod} B)_{\mathrm{rig} / A}$ and let $\left(N, \rho_{N}\right) \in \mathrm{D}_{\mathrm{f}}^{\mathrm{b}}(\operatorname{Mod} C)_{\mathrm{rig} / B}$. Assume condition (iii) of Theorem 4.11 holds. Then the complex $M \otimes_{B}^{\mathrm{L}} N \in \mathrm{D}(\operatorname{Mod} C)$ has a unique rigidifying isomorphism

$$
\rho: M \otimes_{B}^{\mathrm{L}} N \stackrel{\simeq}{\longrightarrow} \operatorname{Sq}_{C / A}\left(M \otimes_{B}^{\mathrm{L}} N\right),
$$

such that the diagram

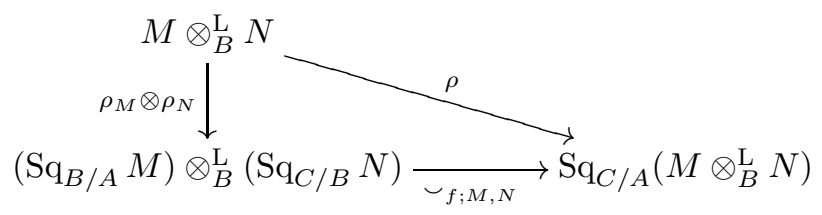

is commutative.

Definition 4.14. In the situation of Corollary 4.13, the induced rigidifying isomorphism of $M \otimes_{B}^{\mathrm{L}} N$ is denoted by $\rho_{M} \otimes \rho_{N}$. In addition we define

$$
\left(M, \rho_{M}\right) \otimes_{B}^{\mathrm{L}}\left(N, \rho_{N}\right):=\left(M \otimes_{B}^{\mathrm{L}} N, \rho_{M} \otimes \rho_{N}\right) \in \mathrm{D}_{\mathrm{f}}^{\mathrm{b}}(\operatorname{Mod} C)_{\operatorname{rig} / A} .
$$

Corollary 4.15. Let $f^{*}: B \rightarrow C$ be a homomorphism between essentially finite type A-algebras, let $\phi:\left(M_{1}, \rho_{M_{1}}\right) \rightarrow\left(M_{2}, \rho_{M_{2}}\right)$ be a morphism in $\mathrm{D}_{\mathrm{f}}^{\mathrm{b}}(\operatorname{Mod} B)_{\mathrm{rig} / A}$, and let $\psi:\left(N_{1}, \rho_{N_{1}}\right) \rightarrow\left(N_{2}, \rho_{N_{2}}\right)$ be a morphism $\mathrm{D}_{\mathrm{f}}^{\mathrm{b}}(\operatorname{Mod} C)_{\mathrm{rig} / B}$. Assume conditions (iii) of Theorem 4.11 hold with respect to the two pairs of complexes $\left(M_{1}, N_{1}\right)$ and $\left(M_{2}, N_{2}\right)$, so that the rigidifying isomorphisms $\rho_{M_{1}} \otimes \rho_{N_{1}}$ and $\rho_{M_{2}} \otimes \rho_{N_{2}}$ exist. Then the morphism

$$
\phi \otimes \psi: M_{1} \otimes_{B}^{\mathrm{L}} N_{1} \rightarrow M_{2} \otimes_{B}^{\mathrm{M}} N_{2}
$$

is a rigid morphism over $C$ relative to $A$.

Proof. This is due to the functoriality of the cup product; see Lemma 4.9.

\section{Rigidity AND Finite HOMOMORPhismS}

In this section we introduce the inverse image operation for rigid complexes with respect to a finite ring homomorphism.

Definition 5.1. Let $f^{*}: A \rightarrow B$ be a ring homomorphism.

(1) Define a functor $f^{b}: \mathrm{D}(\operatorname{Mod} A) \rightarrow \mathrm{D}(\operatorname{Mod} B)$ by $f^{b} M:=\operatorname{RHom}_{A}(B, M)$. 
(2) Given $M \in \mathrm{D}(\operatorname{Mod} A)$ let $\operatorname{Tr}_{f ; M}^{b}: f^{b} M \rightarrow M$ be the morphism $\phi \mapsto \phi(1)$. This becomes a morphism of functors $\operatorname{Tr}_{f}^{b}: f_{*} f^{b} \rightarrow \mathbf{1}_{\mathrm{D}(\operatorname{Mod} A)}$.

Observe that if $g^{*}: B \rightarrow C$ is another ring homomorphism, then adjunction gives rise to a canonical isomorphism

$$
g^{b} f^{b} M=\operatorname{RHom}_{B}\left(C, \operatorname{RHom}_{A}(B, M)\right) \cong \operatorname{RHom}_{A}(C, M)=(f \circ g)^{b} M .
$$

Theorem 5.3. Let $A$ be a noetherian ring, let $B$ and $C$ be essentially finite type $A$-algebras, and let $f^{*}: B \rightarrow C$ be a finite $A$-algebra homomorphism. Suppose we are given a rigid complex $(M, \rho) \in \mathrm{D}_{\mathrm{f}}^{\mathrm{b}}(\operatorname{Mod} B)_{\mathrm{rig} / A}$, such that $f^{\mathrm{b}} M$ has finite flat dimension over $A$.

(1) The complex $f^{b} M \in \mathrm{D}_{\mathrm{f}}^{\mathrm{b}}(\operatorname{Mod} C)$ has an induced rigidifying isomorphism

$$
f^{b}(\rho): f^{b} M \stackrel{\simeq}{\longrightarrow} \operatorname{Sq}_{C / A} f^{b} M \text {. }
$$

The rigid complex $f^{b}(M, \rho):=\left(f^{b} M, f^{b}(\rho)\right)$ depends functorially on $(M, \rho)$.

(2) Suppose $g^{*}: C \rightarrow D$ is another finite homomorphism, and $(f \circ g)^{b} M$ has finite flat dimension over $A$. Then under the isomorphism $(f \circ g)^{b} M \cong$ $g^{b} f^{b} M$ of (5.2) one has

$$
g^{b}\left(f^{b}(\rho)\right)=(f \circ g)^{b}(\rho) .
$$

(3) The morphism $\operatorname{Tr}_{f ; M}^{b}: f^{b} M \rightarrow M$ is a rigid trace morphism relative to $A$.

For the proof we will need a lemma. The catch in this lemma is that the complex $P$ of a flat $A$-module is bounded below, not above.

Lemma 5.4. Let $P$ and $N$ be bounded below complexes of $A$-modules. Assume that each $P^{i}$ is a flat $A$-module, and that $N$ has finite flat dimension over $A$. Then the canonical morphism $P \otimes_{A}^{\mathrm{L}} N \rightarrow P \otimes_{A} N$ in $\mathrm{D}(\operatorname{Mod} A)$ is an isomorphism.

Proof. Choose a bounded flat resolution $Q \rightarrow N$ over $A$. We have to show that $P \otimes_{A} Q \rightarrow P \otimes_{A} N$ is a quasi-isomorphism. Let $L$ be the cone of $Q \rightarrow N$. It is enough to show that the complex $P \otimes_{A} L$ is acyclic. We note that $L$ is a bounded below acyclic complex and $P$ is a bounded below complex of flat modules. To prove that $\mathrm{H}^{i}\left(P \otimes_{A} L\right)=0$ for any given $i$ we might as well replace $P$ with a truncation $P^{\prime}:=\left(\cdots \rightarrow P^{j_{1}-1} \rightarrow P^{j_{1}} \rightarrow 0 \rightarrow \cdots\right)$ for $j_{1} \gg i$. Now $P^{\prime}$ is K-flat, so $P^{\prime} \otimes_{A} L$ is acyclic.

Proof of Theorem 5.3. (1) Let's pick a semi-free DG algebra resolution $A \rightarrow \tilde{B} \rightarrow B$ of $A \rightarrow B$. Next let's pick a K-projective DG algebra resolution $\tilde{B} \rightarrow \tilde{C} \rightarrow C$ of $\tilde{B} \rightarrow C$, such that und $\tilde{C} \cong \bigoplus_{i=-\infty}^{0}$ und $\tilde{B}[-i]^{\mu_{i}}$ with finite multiplicities $\mu_{i}$; see Proposition 1.7(3). Choose a bounded above semi-free resolution $P^{\prime} \rightarrow M$ over $\tilde{B}$. Since $M$ has finite flat dimension over $A$ it follows that for $i \ll 0$ the truncated DG $\tilde{B}$-module $P:=\tau^{\geq i} P^{\prime}$ is a bounded complex of flat $A$-modules, and also $P \cong M$ in $\tilde{\mathrm{D}}(\operatorname{DGMod} \tilde{B})$.

We have an isomorphism $\operatorname{Hom}_{\tilde{B}}(\tilde{C}, P) \cong \operatorname{RHom}_{B}(C, M)=f^{b} M$ in $\tilde{\mathrm{D}}(\operatorname{DGMod} \tilde{C})$, and an isomorphism

$$
\operatorname{Hom}_{\tilde{B} \otimes_{A} \tilde{B}}\left(\tilde{C} \otimes_{A} \tilde{C}, P \otimes_{A} P\right) \cong \operatorname{RHom}_{\tilde{B} \otimes_{A} \tilde{B}}\left(\tilde{C} \otimes_{A} \tilde{C}, M \otimes_{A}^{\mathrm{L}} M\right)
$$

in $\tilde{\mathrm{D}}\left(\mathrm{DGMod} \tilde{C} \otimes_{A} \tilde{C}\right)$. Because the multiplicities $\mu_{i}$ are finite and $P$ is bounded, the obvious DG module homomorphism

$$
\operatorname{Hom}_{\tilde{B}}(\tilde{C}, P) \otimes_{A} \operatorname{Hom}_{\tilde{B}}(\tilde{C}, P) \rightarrow \operatorname{Hom}_{\tilde{B} \otimes_{A} \tilde{B}}\left(\tilde{C} \otimes_{A} \tilde{C}, P \otimes_{A} P\right)
$$


is bijective. Now $\operatorname{Hom}_{\tilde{B}}(\tilde{C}, P)$ is a bounded below complex of flat $A$-modules, which also has finite flat dimension over $A$. Therefore by Lemma 5.4 we obtain

$$
\operatorname{Hom}_{\tilde{B}}(\tilde{C}, P) \otimes_{A} \operatorname{Hom}_{\tilde{B}}(\tilde{C}, P) \cong \operatorname{RHom}_{B}(C, M) \otimes_{A}^{\mathrm{L}} \operatorname{RHom}_{B}(C, M)
$$

in $\tilde{\mathrm{D}}\left(\mathrm{DGMod} \tilde{C} \otimes_{A} \tilde{C}\right)$. We conclude that there is a functorial isomorphism

$$
\operatorname{RHom}_{B}(C, M) \otimes_{A}^{\mathrm{L}} \operatorname{RHom}_{B}(C, M) \cong \operatorname{RHom}_{\tilde{B} \otimes_{A} \tilde{B}}\left(\tilde{C} \otimes_{A} \tilde{C}, M \otimes_{A}^{\mathrm{L}} M\right)
$$

in $\tilde{\mathrm{D}}\left(\mathrm{DGMod} \tilde{C} \otimes_{A} \tilde{C}\right)$. (If $A$ is a field we may disregard the previous sentences, and just take $\tilde{B}:=B$ and $\tilde{C}:=C$.) We thus have a sequence of isomorphisms in $\mathrm{D}(\operatorname{Mod} C)$ :

$$
\begin{aligned}
\operatorname{Sq}_{C / A} f^{b} M & =\operatorname{RHom}_{\tilde{C} \otimes_{A} \tilde{C}}\left(C, \operatorname{RHom}_{B}(C, M) \otimes_{A}^{\mathrm{L}} \operatorname{RHom}_{B}(C, M)\right) \\
& \cong \operatorname{RHom}_{\tilde{C} \otimes_{A} \tilde{C}}\left(C, \operatorname{RHom}_{\tilde{B} \otimes_{A} \tilde{B}}\left(\tilde{C} \otimes_{A} \tilde{C}, M \otimes_{A}^{\mathrm{L}} M\right)\right) \\
& \cong \operatorname{RHom}_{\tilde{B} \otimes_{A} \tilde{B}}\left(C, M \otimes_{A}^{\mathrm{L}} M\right) \\
& \cong \operatorname{RHom}_{B}\left(C, \operatorname{RHom}_{\tilde{B} \otimes_{A} \tilde{B}}\left(B, M \otimes_{A}^{\mathrm{L}} M\right)\right)=f^{b} \operatorname{Sq}_{B / A} M,
\end{aligned}
$$

where the isomorphism marked $\diamond$ is by (5.5), and the isomorphisms $\ddagger$ come from the Hom-tensor adjunction formula. The rigidifying isomorphism we want is the composition of

$$
f^{b}(\rho): f^{b} M \rightarrow f^{b} \mathrm{Sq}_{B / A} M
$$

with the isomorphism $\alpha_{f}: f^{b} \mathrm{Sq}_{B / A} M \stackrel{\simeq}{\longrightarrow} \mathrm{Sq}_{C / A} f^{b} M$ we get from (5.6).

(2) Because of adjunction identities the diagram

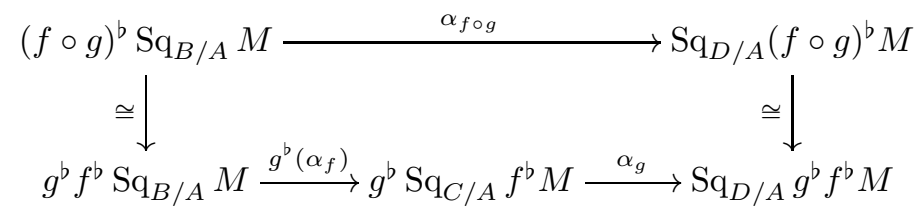

in which the vertical arrows are the isomorphisms (5.2), is commutative. Therefore $g^{b}\left(f^{b}(\rho)\right)=(f \circ g)^{b}(\rho)$.

(3) Consider the diagram

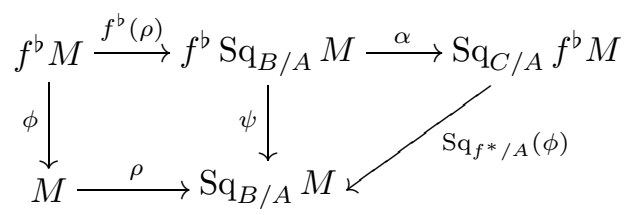

in which $\phi:=\operatorname{Tr}_{f ; M}^{b}, N:=\operatorname{Sq}_{B / A} M$ and $\psi:=\operatorname{Tr}_{f ; N}^{b}$. Since $\operatorname{Tr}_{f}: f_{*} f^{b} \rightarrow \mathbf{1}$ is a natural transformation, the square portion on diagram (5.8) is commutative.

As for the triangle portion of the diagram, according to Theorem 2.3 the morphism

$$
\mathrm{Sq}_{f^{*} / A}(\phi): \mathrm{Sq}_{C / A} f^{b} M \rightarrow \mathrm{Sq}_{B / A} M
$$

is just

$$
\left(f^{*}, \phi \otimes \phi\right): \operatorname{RHom}_{\tilde{C} \otimes_{A} \tilde{C}}\left(C,\left(f^{b} M\right) \otimes_{A}^{\mathrm{L}}\left(f^{b} M\right)\right) \rightarrow \operatorname{RHom}_{\tilde{B} \otimes_{A} \tilde{B}}\left(B, M \otimes_{A}^{\mathrm{L}} M\right) .
$$


Now going over the various isomorphisms in equation (5.6) we see that every one of them commutes with the obvious morphisms to $\operatorname{RHom}_{\tilde{B} \otimes_{A} \tilde{B}}\left(B, M \otimes_{A}^{\mathrm{L}} M\right)$. Therefore the triangle portion of diagram (5.8) is commutative, and so $\operatorname{Tr}_{f ; M}^{b}$ is a rigid trace morphism.

Suppose $B \rightarrow C$ is a ring homomorphism, $M \in \mathrm{D}(\operatorname{Mod} B)$ and $N \in \mathrm{D}(\operatorname{Mod} C)$. Adjunction gives rise to a canonical isomorphism

$$
\operatorname{RHom}_{B}(N, M) \cong \operatorname{RHom}_{C}\left(N, \operatorname{RHom}_{B}(C, M)\right)=\operatorname{RHom}_{C}\left(N, f^{\mathrm{b}} M\right) .
$$

Given a morphism $\psi: N \rightarrow f^{b} M$ in $\mathrm{D}(\operatorname{Mod} C)$, the corresponding morphism in $\mathrm{D}(\operatorname{Mod} B)$ is $\operatorname{Tr}_{f ; M}^{b} \circ \psi: N \rightarrow M$.

Definition 5.9. Let $f^{*}: B \rightarrow C$ be a ring homomorphism, $M \in \mathrm{D}(\operatorname{Mod} B)$ and $N \in \mathrm{D}(\operatorname{Mod} C)$. A morphism $\phi: N \rightarrow M$ in $\mathrm{D}(\operatorname{Mod} B)$ is called a nondegenerate trace morphism if the corresponding morphism $N \rightarrow f^{b} M$ in $\mathrm{D}(\operatorname{Mod} C)$ is an isomorphism.

Proposition 5.10. Let $f^{*}: B \rightarrow C$ be a finite homomorphism between two essentially finite type A-algebras, let $\left(M, \rho_{M}\right) \in \mathrm{D}_{\mathrm{f}}^{\mathrm{b}}(\operatorname{Mod} B)_{\mathrm{rig} / A}$ and $\left(N, \rho_{N}\right) \in$ $\mathrm{D}_{\mathrm{f}}^{\mathrm{b}}(\operatorname{Mod} C)_{\mathrm{rig} / A}$. Assume $N \cong f^{\mathrm{b}} M$ and $\operatorname{Hom}_{\mathrm{D}(\operatorname{Mod} C)}(N, N)=C$. Then there exists a unique nondegenerate rigid trace morphism $\phi:\left(N, \rho_{N}\right) \rightarrow\left(M, \rho_{M}\right)$ over $B$ relative to $A$.

Proof. First note that in this case a morphism $N \rightarrow M$ is a nondegenerate trace morphism if and only if the corresponding morphism $N \rightarrow f^{b} M$ is a basis of the rank 1 free $C$-module $\operatorname{Hom}_{\mathrm{D}(\operatorname{Mod} C)}\left(N, f^{b} M\right)$.

Because $N \cong f^{b} M$ this has finite flat dimension over $A$, so Theorem 5.3(1) applies, and the rigid complex $f^{b}\left(M, \rho_{M}\right)$ exists. Pick any isomorphism $\psi: N \stackrel{\simeq}{\longrightarrow}$ $f^{b} M$. Then $\operatorname{Hom}_{\mathrm{D}(\operatorname{Mod} C)}\left(N, f^{b} M\right)$ is a free $C$-module with basis $\psi$. Since $\operatorname{Sq}_{C / A}(\psi)$ : $\mathrm{Sq}_{C / A} N \rightarrow \mathrm{Sq}_{C / A} f^{b} M$ is also an isomorphism, it follows that $\operatorname{Sq}_{C / A}(\psi)=u f^{b}\left(\rho_{M}\right)$ $\circ \psi$ for some unique invertible $u \in C$. Then $u^{-1} \psi:\left(N, \rho_{N}\right) \rightarrow f^{b}\left(M, \rho_{M}\right)$ is a rigid isomorphism. By Theorem 5.3(2) the morphism

$$
\phi:=\operatorname{Tr}_{f ; M}^{b} \circ u^{-1} \psi:\left(N, \rho_{N}\right) \rightarrow\left(M, \rho_{M}\right)
$$

is a nondegenerate rigid trace morphism.

Finally, since $u^{-1} \psi$ is the unique rigid isomorphism $\left(N, \rho_{N}\right) \rightarrow f^{\mathrm{b}}\left(M, \rho_{M}\right)$ it follows that $\phi$ is the unique nondegenerate rigid trace morphism $\left(N, \rho_{N}\right) \rightarrow$ $\left(M, \rho_{M}\right)$.

Corollary 5.11. In the situation of Theorem 5.3, assume $\operatorname{Hom}_{\mathrm{D}(\operatorname{Mod} C)}\left(f^{b} M, f^{b} M\right)$ $=C$. Then $\operatorname{Tr}_{f ; M}^{b}$ is the unique nondegenerate rigid trace morphism $f^{b}\left(M, \rho_{M}\right)$ $\rightarrow\left(M, \rho_{M}\right)$.

Proof. Take $\left(N, \rho_{N}\right):=f^{b}\left(M, \rho_{M}\right)$ in Proposition 5.10.

Example 5.12. Suppose $f^{*}: A \rightarrow B$ is a finite flat ring homomorphism. Consider the rigid complex $(N, \rho):=f^{b}\left(A, \rho_{A}^{\mathrm{tau}}\right) \in \mathrm{D}_{\mathrm{f}}^{\mathrm{b}}(\operatorname{Mod} B)_{\mathrm{rig} / A}$. Since $\mathrm{Sq}_{B / A} N \cong N$ it follows that

$$
\mathrm{Sq}_{B / A} N=\operatorname{Hom}_{B \otimes_{A} B}\left(B, N \otimes_{A} N\right) \subset N \otimes_{A} N
$$

and in this way we may view $\rho$ as an $A$-linear homomorphism $\rho: N \rightarrow N \otimes_{A} N$. It is not too hard to show (using Corollary 5.11) that $\rho$ makes $N$ into a coassociative 
cocommutative coalgebra over $A$, with counit $\operatorname{Tr}_{f ; A}^{b}: N \rightarrow A$. In fact, the coalgebra $N$ is precisely the $A$-linear dual of the $A$-algebra $B$.

\section{Rigidity AND ESSENTIALLY SMOOTH HOMOMORPHISMS}

In this section we introduce the inverse image operation for rigid complexes with respect to an essentially smooth ring homomorphism.

We shall need the following easy fact.

Lemma 6.1. Suppose $B=\prod_{i=1}^{m} B_{i}$, i.e. $\operatorname{Spec} B=\coprod_{i=1}^{m}$ Spec $B_{i}$. Then the functor $N \mapsto \prod_{i}\left(B_{i} \otimes_{B} N\right)$ is an equivalence $\mathrm{D}(\operatorname{Mod} B) \rightarrow \prod_{i} \mathrm{D}\left(\operatorname{Mod} B_{i}\right)$.

Definition 6.2. Suppose $f^{*}: A \rightarrow B$ is an essentially smooth homomorphism between noetherian rings. Let $\operatorname{Spec} B=\coprod_{i} \operatorname{Spec} B_{i}$ be the (finite) decomposition of Spec $B$ into connected components. For each $i$ the $B_{i}$-module $\Omega_{B_{i} / A}^{1}$ is projective of constant rank, say $n_{i}$. Given $M \in \mathrm{D}(\operatorname{Mod} A)$ define

$$
f^{\sharp} M:=\prod_{i}\left(\Omega_{B_{i} / A}^{n_{i}}\left[n_{i}\right] \otimes_{A} M\right) .
$$

This is a functor $f^{\sharp}: \mathrm{D}(\operatorname{Mod} A) \rightarrow \mathrm{D}(\operatorname{Mod} B)$.

Note that if $f^{*}: A \rightarrow B$ is essentially étale, then one simply has $f^{\sharp} M=B \otimes_{A} M$.

Theorem 6.3. Let $A$ be a noetherian ring, let $B$ and $C$ be essentially finite type $A$-algebras, and let $f^{*}: B \rightarrow C$ be an essentially smooth $A$-algebra homomorphism. Let $(L, \rho) \in \mathrm{D}_{\mathrm{f}}^{\mathrm{b}}(\operatorname{Mod} B)_{\text {rig } / A}$.

(1) The complex $f^{\sharp} L$ has an induced rigidifying isomorphism

$$
f^{\sharp}(\rho): f^{\sharp} L \stackrel{\simeq}{\longrightarrow} \mathrm{Sq}_{C / A} f^{\sharp} L \text {. }
$$

We get a functor $f^{\sharp}: \mathrm{D}_{\mathrm{f}}^{\mathrm{b}}(\operatorname{Mod} B)_{\mathrm{rig} / A} \rightarrow \mathrm{D}_{\mathrm{f}}^{\mathrm{b}}(\operatorname{Mod} C)_{\mathrm{rig} / A}$.

(2) Let $\left(B, \rho_{B}^{\mathrm{tau}}\right)$ be the tautological rigid complex. Then under the standard isomorphism $f^{\sharp} L \cong L \otimes \otimes_{B}^{\mathrm{L}} f^{\sharp} B$ one has $f^{\sharp}(\rho)=\rho \otimes f^{\sharp}\left(\rho_{B}^{\text {tau }}\right)$.

(3) Let $g^{*}: C \rightarrow D$ be an essentially smooth homomorphism. Then under the isomorphism $(f \circ g)^{\sharp} L \cong g^{\sharp} f^{\sharp} L$ of Proposition 3.4 one has $(f \circ g)^{\sharp}(\rho)=$ $g^{\sharp} f^{\sharp}(\rho)$.

Proof. (1) In view of Lemma 6.1 we might as well assume $\Omega_{C / B}^{1}$ has constant rank $m$. There are ring homomorphisms $\mathrm{p}_{1}^{*}, \mathrm{p}_{2}^{*}: C \rightarrow C \otimes_{B} C$, namely $\mathrm{p}_{1}^{*}(c):=c \otimes 1$ and $\mathrm{p}_{2}^{*}(c):=1 \otimes c$. These induce module homomorphisms $\mathrm{p}_{i}^{*}: \Omega_{C / B}^{m} \rightarrow \Omega_{\left(C \otimes_{B} C\right) / B}^{m}$, and the resulting homomorphism

$$
\Omega_{C / B}^{m} \otimes_{B} \Omega_{C / B}^{m} \rightarrow \Omega_{\left(C \otimes_{B} C\right) / B}^{2 m}, \gamma_{1} \otimes \gamma_{2} \mapsto \mathrm{p}_{1}^{*}\left(\gamma_{1}\right) \wedge \mathrm{p}_{2}^{*}\left(\gamma_{2}\right)
$$

is bijective. So we can interpret Theorem $3.14(3)$ as a canonical rigidifying isomorphism for the complex $\Omega_{C / B}^{m}[m]$ relative to $B$, which we denote by $\rho_{\Omega}$. Thus we obtain an object

$$
\left(\Omega_{C / B}^{m}[m], \rho_{\Omega}\right) \in \mathrm{D}_{\mathrm{f}}^{\mathrm{b}}(\operatorname{Mod} C)_{\mathrm{rig} / B} .
$$

Now using Theorem 4.11, under its condition (iii)(a), and Definition 4.14, we can define the rigidifying isomorphism $f^{\sharp}(\rho):=\rho \otimes \rho_{\Omega}$. Corollary 4.15 guarantees functoriality.

(2) This assertion is clear from part (1). 
(3) We may assume $\Omega_{D / C}^{1}$ has constant rank $n$. Let's introduce temporary notation $C^{\mathrm{e} / B}:=C \otimes_{B} C$ and $\mathrm{E}(B, C):=\operatorname{Ext}_{C^{\mathrm{e} / B}}^{m}\left(C, \Omega_{C^{\mathrm{e} / B} / B}^{2 m}\right)$, so that

$$
\mathrm{Sq}_{C / B}\left(\Omega_{C / B}^{m}[m]\right)=\mathrm{E}(B, C)[m] .
$$

Likewise for $C \rightarrow D$ and $B \rightarrow D$.

Using part (2) of this theorem it suffices to prove that

$$
(f \circ g)^{\sharp}\left(\rho_{B}^{\mathrm{tau}}\right)=f^{\sharp}\left(\rho_{B}^{\mathrm{tau}}\right) \otimes g^{\sharp}\left(\rho_{C}^{\mathrm{tau}}\right)
$$

as rigidifying isomorphisms for $\Omega_{D / B}^{m+n}[m+n]=g^{\sharp} f^{\sharp} B$ relative to $B$. In other words, we have to prove that the diagram of isomorphisms

$$
\begin{gathered}
\Omega_{C / B}^{m} \otimes_{C} \Omega_{D / C}^{n} \longrightarrow \\
f^{\sharp}\left(\rho_{B}^{\mathrm{tau}}\right) \otimes g^{\sharp}\left(\rho_{C}^{\mathrm{tau}}\right){ }_{D / B}^{m+n} \\
\mathrm{E}(B, C) \otimes_{C} \mathrm{E}(C, D) \longrightarrow \mathrm{E}(B, D)
\end{gathered}
$$

in which the top horizontal arrow is from Proposition 3.4, and the bottom horizontal arrow is the isomorphism of Theorem 4.11(2), shifted by $m+n$, is commutative.

Let us define ideals $J:=\operatorname{Ker}\left(C^{\mathrm{e} / B} \rightarrow C\right)$ and $K:=\operatorname{Ker}\left(D^{\mathrm{e} / C} \rightarrow D\right)$. Choose elements $s \in C^{\mathrm{e} / B}$ and $t \in D^{\mathrm{e} / C}$ such that the ideals $J\left[s^{-1}\right]$ and $K\left[t^{-1}\right]$ are generated by regular sequences $\boldsymbol{c}=\left(c_{1}, \ldots, c_{m}\right)$ and $\boldsymbol{d}=\left(d_{1}, \ldots, d_{n}\right)$, respectively. Using the surjection $D^{\mathrm{e} / B} \rightarrow D^{\mathrm{e} / C}$ we lift $t$ to an element of $D^{\mathrm{e} / B}$, which is also denoted by $t$. After possibly replacing $t$ by $s t$ we can assume that $C^{\mathrm{e} / B}\left[s^{-1}\right]$ goes into $D^{\mathrm{e} / B}\left[t^{-1}\right]$. In view of Lemma 3.8 it suffices to verify that the diagram (6.4) commutes after inverting $t$.

Let's write

$$
\mathrm{E}(B, C)\left[s^{-1}\right]:=\operatorname{Ext}_{C^{\mathrm{e} / B}\left[s^{-1}\right]}^{m}\left(C\left[s^{-1}\right], \Omega_{C^{\mathrm{e} / B}\left[s^{-1}\right] / B}^{2 m}\right) .
$$

We can represent it using a Koszul complex:

$$
\mathrm{E}(B, C)\left[s^{-1}\right]=\mathrm{H}^{m} \operatorname{Hom}_{C^{\mathrm{e} / B}\left[s^{-1}\right]}\left(\mathbf{K}\left(C^{\mathrm{e} / B}\left[s^{-1}\right], \boldsymbol{c}\right), \Omega_{C^{\mathrm{e} / B}\left[s^{-1}\right] / B}^{2 m}\right) .
$$

Likewise for $\mathrm{E}(C, D)\left[t^{-1}\right]$ and $\mathrm{E}(B, D)\left[t^{-1}\right]$, using the regular sequences $\boldsymbol{d}$ and $(\boldsymbol{c}, \boldsymbol{d})$, respectively. Now let's return to the proof of Theorem 4.11(2) and track all the isomorphisms there. But this time we have flat algebra homomorphisms $B \rightarrow C \rightarrow D$ (instead of $A \rightarrow B \rightarrow C$ ), so there is no need to take DG algebra resolutions. On the other hand there are some localizations to remember. Thus, instead of $\tilde{B} \otimes_{A} \tilde{B}$ we now have $C^{\mathrm{e} / B}\left[s^{-1}\right]$, instead of $B$ we now have $C\left[s^{-1}\right]$, etc. Most importantly, the RHom's are calculated using Koszul complexes, so elements of $\mathrm{E}(B, C)\left[s^{-1}\right]$, etc., can be represented as generalized fractions. We see that the isomorphism

$$
\mathrm{E}(B, C)\left[s^{-1}\right] \otimes_{C\left[s^{-1}\right]} \mathrm{E}(C, D)\left[t^{-1}\right] \stackrel{\simeq}{\longrightarrow} \mathrm{E}(B, D)\left[t^{-1}\right]
$$

that we get from Theorem 4.11(2) sends

$$
\left[\begin{array}{c}
\mathrm{d}(\boldsymbol{c}) \wedge \mathrm{p}_{2}^{*}(\gamma) \\
\boldsymbol{c}
\end{array}\right] \otimes\left[\begin{array}{c}
\mathrm{d}(\boldsymbol{d}) \wedge \mathrm{p}_{2}^{*}(\delta) \\
\boldsymbol{d}
\end{array}\right] \mapsto\left[\begin{array}{c}
\mathrm{d}(\boldsymbol{c}, \boldsymbol{d}) \wedge \mathrm{p}_{2}^{*}(\gamma \wedge \delta) \\
(\boldsymbol{c}, \boldsymbol{d})
\end{array}\right] .
$$

Here $\gamma \in \Omega_{C / B}^{m}$ and $\delta \in \Omega_{D / C}^{n}$ are arbitrary elements. Comparing this to Lemma $3.8(1)$ we conclude that indeed the diagram (6.4), localized, is commutative. 
Definition 6.5. Let $A$ be a noetherian ring, and let $f^{*}: A \rightarrow A^{\prime}$ be an essentially étale ring homomorphism. For $M \in \mathrm{D}(\operatorname{Mod} A)$ let $\mathrm{q}_{f ; M}^{\sharp}: M \rightarrow f^{\sharp} M=A^{\prime} \otimes_{A} M$ be the morphism $m \mapsto 1 \otimes m$. As $M$ varies this becomes a functorial morphism $\mathrm{q}_{f}^{\sharp}: \mathbf{1}_{\mathrm{D}(\operatorname{Mod} A)} \rightarrow f_{*} f^{\sharp}$.

In the situation of the definition above, given $M^{\prime} \in \mathrm{D}\left(\operatorname{Mod} A^{\prime}\right)$, there is a canonical bijection

$$
\operatorname{Hom}_{\mathrm{D}(\operatorname{Mod} A)}\left(M, M^{\prime}\right) \cong \operatorname{Hom}_{\mathrm{D}\left(\operatorname{Mod} A^{\prime}\right)}\left(f^{\sharp} M, M^{\prime}\right), \phi \mapsto \mathbf{1} \otimes \phi .
$$

In particular, for $M^{\prime}:=f^{\sharp} M$, the morphism $q_{f ; M}^{\sharp}$ corresponds to the identity $\mathbf{1}_{M^{\prime}}$.

Definition 6.6. Let $A$ be a noetherian ring, let $A \rightarrow A^{\prime}$ be an essentially étale ring homomorphism, and let $M \in \mathrm{D}(\operatorname{Mod} A)$ and $M^{\prime} \in \mathrm{D}\left(\operatorname{Mod} A^{\prime}\right)$. A morphism $\phi: M \rightarrow M^{\prime}$ in $\mathrm{D}(\operatorname{Mod} A)$ is called a nondegenerate localization morphism if the induced morphism $1 \otimes \phi: A^{\prime} \otimes_{A} M \rightarrow M^{\prime}$ is an isomorphism.

Definition 6.7. Let $A$ be a noetherian ring, let $B$ and $B^{\prime}$ be essentially finite type $A$-algebras, let $f^{*}: B \rightarrow B^{\prime}$ be an essentially étale $A$-algebra homomorphism, let $(M, \rho) \in \mathrm{D}_{\mathrm{f}}^{\mathrm{b}}(\operatorname{Mod} B)_{\mathrm{rig} / A}$ and let $\left(M^{\prime}, \rho^{\prime}\right) \in \mathrm{D}_{\mathrm{f}}^{\mathrm{b}}\left(\operatorname{Mod} B^{\prime}\right)_{\mathrm{rig} / A}$. A rigid localization morphism is a morphism $\phi: M \rightarrow M^{\prime}$ in $\mathrm{D}(\operatorname{Mod} B)$, such that the induced morphism $1 \otimes \phi: f^{\sharp}(M, \rho) \rightarrow\left(M^{\prime}, \rho^{\prime}\right)$ is a rigid morphism over $B^{\prime}$ relative to $A$, in the sense of Definition 4.3.

Proposition 6.8. Let $A$ be a noetherian ring, let $B$ and $B^{\prime}$ be essentially finite type $A$-algebras, let $f^{*}: B \rightarrow B^{\prime}$ be an essentially étale $A$-algebra homomorphism, and let $(M, \rho) \in \mathrm{D}_{\mathrm{f}}^{\mathrm{b}}(\operatorname{Mod} B)_{\mathrm{rig} / A}$. Define $\left(M^{\prime}, \rho^{\prime}\right):=f^{\sharp}(M, \rho)$. Then:

(1) The morphism $\mathrm{q}_{f ; M}^{\sharp}: M \rightarrow M^{\prime}$ is a nondegenerate rigid localization morphism.

(2) Moreover, if $\operatorname{RHom}_{B}(M, M)=B$, then $\mathrm{q}_{f ; M}^{\sharp}$ is the unique nondegenerate rigid localization morphism $M \rightarrow M^{\prime}$.

Proof. (1) Since the corresponding morphism $M^{\prime} \rightarrow M^{\prime}$ is the identity automorphism of $M^{\prime}$, it is certainly rigid and nondegenerate.

(2) Here we have $\operatorname{Hom}_{\mathrm{D}\left(\operatorname{Mod} B^{\prime}\right)}\left(M^{\prime}, M^{\prime}\right)=B^{\prime}$. The uniqueness of $\mathrm{q}_{f ; M}^{\sharp}$ is proved as in Corollary 5.11.

Theorem 6.9 (Base change). Let $A$ be a noetherian ring, let $B, B^{\prime}$ and $C$ be essentially finite type $A$-algebras, and let $f^{*}: B \rightarrow C$ and $g^{*}: B \rightarrow B^{\prime}$ be homomorphisms of $A$-algebras, with $g^{*}$ a localization. Define $C^{\prime}:=B^{\prime} \otimes_{B} C$, so there are induced homomorphisms $f^{\prime *}: B^{\prime} \rightarrow C^{\prime}$ and $h^{*}: C \rightarrow C^{\prime}$, with $h^{*}$ a localization; $c f$. the diagram below. Let $\left(M, \rho_{M}\right) \in \mathrm{D}_{\mathrm{f}}^{\mathrm{b}}(\operatorname{Mod} B)_{\operatorname{rig} / A}$, let $\left(N, \rho_{N}\right) \in \mathrm{D}_{\mathrm{f}}^{\mathrm{b}}(\operatorname{Mod} C)_{\operatorname{rig} / A}$, and suppose

$$
\phi:\left(N, \rho_{N}\right) \rightarrow\left(M, \rho_{M}\right)
$$

is a rigid trace morphism over $B$ relative to $A$. Define $M^{\prime}:=g^{\sharp} M$ and $N^{\prime}:=$ $h^{\sharp} N$. There is a morphism $\phi^{\prime}: N^{\prime} \rightarrow M^{\prime}$ in $\mathrm{D}\left(\operatorname{Mod} B^{\prime}\right)$ obtained by composing the canonical isomorphism $N^{\prime}=C^{\prime} \otimes_{C} N \cong B^{\prime} \otimes_{B} N=g^{\sharp} N$ with $g^{\sharp}(\phi): g^{\sharp} N \rightarrow$ $g^{\sharp} M=M^{\prime}$. Then

$$
\phi^{\prime}:\left(N^{\prime}, h^{\sharp}\left(\rho_{N}\right)\right) \rightarrow\left(M^{\prime}, g^{\sharp}\left(\rho_{M}\right)\right)
$$


is a rigid trace morphism over $B^{\prime}$ relative to $A$.
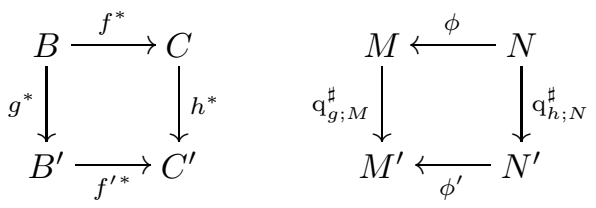

Observe there is no particular assumption on $f^{*}$, and $\phi$ is not assumed to be nondegenerate. For the proof we shall need a few lemmas.

Choose a K-flat DG algebra resolution $A \rightarrow \tilde{B} \rightarrow B$ of $A \rightarrow B$, and a K-flat DG algebra resolution $\tilde{B} \rightarrow \tilde{B}^{\prime} \rightarrow B^{\prime}$ of $\tilde{B} \rightarrow B^{\prime}$; see Proposition 1.7. (If $A \rightarrow B$ and $B \rightarrow B^{\prime}$ are flat, then we may just take $\tilde{B}:=B$ and $\tilde{B}^{\prime}:=B^{\prime}$.) Define a morphism

$$
\lambda_{M}: \mathrm{Sq}_{B / A} M \rightarrow \mathrm{Sq}_{B^{\prime} / A} M^{\prime}
$$

in $\mathrm{D}(\operatorname{Mod} B)$ by composing the following morphisms:

$$
\begin{aligned}
\mathrm{Sq}_{B / A} M & =\operatorname{RHom}_{\tilde{B} \otimes_{A} \tilde{B}}\left(B, M \otimes_{A}^{\mathrm{L}} M\right) \\
& \stackrel{(\mathbf{1}, \mathrm{q} \otimes \mathrm{q})}{\longrightarrow} \operatorname{RHom}_{\tilde{B} \otimes_{A} \tilde{B}}\left(B, M^{\prime} \otimes_{A}^{\mathrm{L}} M^{\prime}\right) \\
& \stackrel{\simeq}{\longrightarrow} \mathrm{RHom}_{\tilde{B}^{\prime} \otimes_{A} \tilde{B}^{\prime}}\left(B^{\prime}, M^{\prime} \otimes_{A}^{\mathrm{L}} M^{\prime}\right) \\
& =\operatorname{Sq}_{B^{\prime} / A} M^{\prime} .
\end{aligned}
$$

In these formulas 1 denotes the identity morphism of $B$, and $\mathrm{q}:=\mathrm{q}_{g ; M}^{\sharp}: M \rightarrow M^{\prime}$. For the notation $(\mathbf{1}, \mathrm{q} \otimes \mathrm{q})$ see text prior to Theorem 2.3. The isomorphism $\diamond$ is by Hom-tensor adjunction with respect to the DG algebra homomorphism $\tilde{B} \otimes_{A} \tilde{B} \rightarrow$ $\tilde{B}^{\prime} \otimes_{A} \tilde{B}^{\prime}$, plus the fact that $B \otimes_{\tilde{B} \otimes_{A} \tilde{B}}\left(\tilde{B}^{\prime} \otimes_{A} \tilde{B}^{\prime}\right) \cong B^{\prime}$.

Lemma 6.11. The morphism $\lambda_{M}$ is independent of the $D G$ algebra resolutions $A \rightarrow \tilde{B} \rightarrow B$ and $\tilde{B} \rightarrow \tilde{B}^{\prime} \rightarrow B^{\prime}$.

Proof. Choose a K-injective resolution $M \otimes_{A}^{\mathrm{L}} M \rightarrow I$ over $\tilde{B} \otimes_{A} \tilde{B}$, and a K-injective resolution $M^{\prime} \otimes_{A}^{\mathrm{L}} M^{\prime} \rightarrow I^{\prime}$ over $\tilde{B}^{\prime} \otimes_{A} \tilde{B}^{\prime}$. Since $\tilde{B}^{\prime} \otimes_{A} \tilde{B}^{\prime}$ is K-flat over $\tilde{B} \otimes_{A} \tilde{B}$ it follows that $I^{\prime}$ is a K-injective $\mathrm{DG} \tilde{B} \otimes_{A} \tilde{B}$-module. Therefore $\mathrm{q} \otimes \mathrm{q}: M \otimes_{A} M \rightarrow$ $M^{\prime} \otimes_{A} M^{\prime}$ extends to a homomorphism $\psi: I \rightarrow I^{\prime}$ in DGMod $\tilde{B} \otimes_{A} \tilde{B}$, uniquely up to homotopy. The morphism $\lambda_{M}$ is represented by

$$
\operatorname{Hom}_{\tilde{B} \otimes_{A} \tilde{B}}(B, I) \stackrel{(\mathbf{1}, \psi)}{\longrightarrow} \operatorname{Hom}_{\tilde{B} \otimes_{A} \tilde{B}}\left(B, I^{\prime}\right) \stackrel{\simeq}{\longrightarrow} \operatorname{Hom}_{\tilde{B}^{\prime} \otimes_{A} \tilde{B}^{\prime}}\left(B^{\prime}, I^{\prime}\right) .
$$

Now the homotopy argument from the proof of Theorem 2.2 can be used.

In the same way we define a morphism $\lambda_{N}: \mathrm{Sq}_{C / A} N \rightarrow \mathrm{Sq}_{C^{\prime} / A} N^{\prime}$ in $\mathrm{D}(\operatorname{Mod} C)$.

Lemma 6.12. The diagram

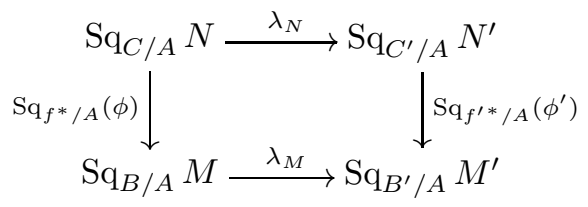

of morphisms in $\mathrm{D}(\operatorname{Mod} B)$ is commutative. 
Proof. Choose a semi-free DG algebra resolution $A \rightarrow \tilde{B} \rightarrow B$ of $A \rightarrow B$. Next choose semi-free DG algebra resolutions $\tilde{B} \stackrel{v}{\rightarrow} \tilde{B}^{\prime} \rightarrow B^{\prime}$ and $\tilde{B} \stackrel{u}{\rightarrow} \tilde{C} \rightarrow C$ of $\tilde{B} \rightarrow B^{\prime}$ and $\tilde{B} \rightarrow C$, respectively. Define $\tilde{C}^{\prime}:=\tilde{B}^{\prime} \otimes_{\tilde{B}} \tilde{C}$. Because $B \rightarrow B^{\prime}$ is flat we get a quasi-isomorphism $\tilde{C}^{\prime} \rightarrow C^{\prime}$, and hence $\tilde{C} \stackrel{w}{\longrightarrow} \tilde{C}^{\prime} \rightarrow C^{\prime}$ and $\tilde{B}^{\prime} \stackrel{u^{\prime}}{\longrightarrow} \tilde{C}^{\prime} \rightarrow C^{\prime}$ are semi-free DG algebra resolutions of $\tilde{C} \rightarrow C^{\prime}$ and $\tilde{B}^{\prime} \rightarrow C^{\prime}$, respectively. By definition of the morphisms $\lambda_{-}$and $\mathrm{Sq}_{-}$the diagram (6.13) is represented by

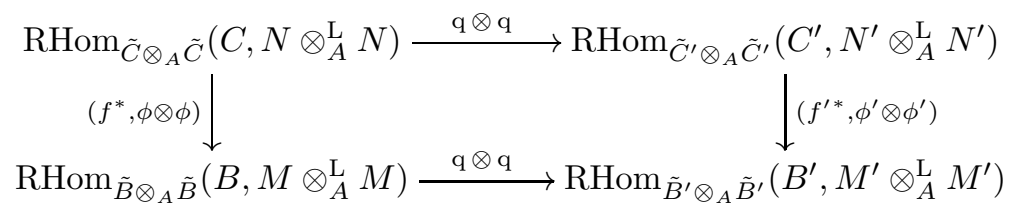

(we are suppressing the adjunction isomorphisms $\diamond$ ). This latter diagram is trivially commutative.

Lemma 6.14. The diagram

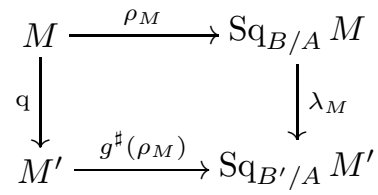

of morphisms in $\mathrm{D}(\operatorname{Mod} B)$ is commutative.

Proof. Recall that $g^{\sharp}\left(\rho_{M}\right)$ was defined to be $g^{\sharp}\left(\rho_{B}^{\mathrm{tau}}\right) \otimes \rho_{M}$, under the isomorphism

$$
\left(\mathrm{Sq}_{B / A} M\right) \otimes_{B}^{\mathrm{L}}\left(\mathrm{Sq}_{B^{\prime} / B} B^{\prime}\right) \stackrel{\smile_{g^{*} ; M, B^{\prime}}^{\longrightarrow}}{\longrightarrow} \mathrm{Sq}_{B^{\prime} / A} M^{\prime} .
$$

But in this situation of a localization $B^{\prime} \otimes_{B} B^{\prime}=B^{\prime}$, and

$$
g^{\sharp}\left(\rho_{B}^{\mathrm{tau}}\right): B^{\prime} \stackrel{\simeq}{\longrightarrow} \mathrm{Sq}_{B^{\prime} / B} B^{\prime}=\mathrm{RHom}_{B^{\prime} \otimes_{B} B^{\prime}}\left(B^{\prime}, B^{\prime} \otimes_{B} B^{\prime}\right)=B^{\prime}
$$

is just the identity. The composed map

$$
\mathrm{Sq}_{B / A} M \stackrel{\mathrm{q}}{\rightarrow}\left(\mathrm{Sq}_{B / A} M\right) \otimes_{B}^{\mathrm{L}} B^{\prime} \stackrel{\smile_{g^{*} ; M, B^{\prime}}}{\longrightarrow} \mathrm{Sq}_{B^{\prime} / A} M^{\prime}
$$

is exactly $\lambda_{M}$.

Proof of Theorem 6.9. Consider the cubic diagram

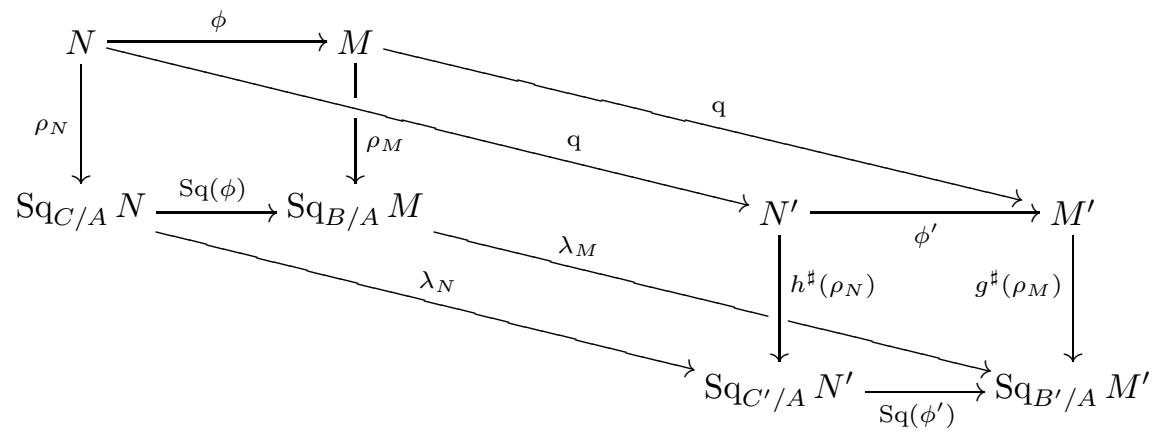

The top face of the diagram is trivially commutative. The right face is commutative by Lemma 6.14 . For the same reason the left face commutes. The bottom face 
commutes by Lemma 6.12. Because $\rho_{M}$ and $\rho_{N}$ are isomorphisms we see that $B^{\prime} \otimes_{B} \mathrm{Sq}_{C / A} N \cong \mathrm{Sq}_{C^{\prime} / A} N^{\prime}$ and $B^{\prime} \otimes_{B} \mathrm{Sq}_{B / A} M \cong \mathrm{Sq}_{B^{\prime} / A} M^{\prime}$, via the morphisms $\lambda_{N}$ and $\lambda_{M}$, respectively. Thus the front face is obtained from the rear face by applying the functor $g^{\sharp}=B^{\prime} \otimes_{B}$ - to it. But the rear face is commutative because $\phi$ is a rigid trace morphism. We conclude that the front face is commutative, which means that $\phi^{\prime}$ is a rigid trace morphism.

Remark 6.15. Theorem 6.9 is true even when $g^{*}$ is essentially étale (not just a localization). However the proof is more complicated, since in this case $B^{\prime} \otimes_{B} B^{\prime}$ is not equal to $B^{\prime}$; cf. Proposition 3.15. In the definition of the morphism $\lambda_{M}$, namely in equation (6.10), one has to replace the isomorphism $\diamond$ with

$$
\begin{aligned}
\operatorname{RHom}_{\tilde{B} \otimes_{A} \tilde{B}}\left(B, M^{\prime} \otimes_{A}^{\mathrm{L}} M^{\prime}\right) & \cong \operatorname{RHom}_{\tilde{B}^{\prime} \otimes_{A} \tilde{B}^{\prime}}\left(B^{\prime} \otimes_{B} B^{\prime}, M^{\prime} \otimes_{A}^{\mathrm{L}} M^{\prime}\right) \\
& \stackrel{(\nu, \mathbf{1} \otimes \mathbf{1})}{\longrightarrow} \operatorname{RHom}_{\tilde{B}^{\prime} \otimes_{A} \tilde{B}^{\prime}}\left(B^{\prime}, M^{\prime} \otimes_{A}^{\mathrm{L}} M^{\prime}\right),
\end{aligned}
$$

where $\nu: B^{\prime} \rightarrow B^{\prime} \otimes_{B} B^{\prime}$ is the canonical homomorphism from Proposition 3.15.

We end the paper with an existence result.

Theorem 6.16. Let $\mathbb{K}$ be a regular noetherian ring of finite Krull dimension, and let $A$ be an essentially finite type $\mathbb{K}$-algebra. Then $A$ has a rigid complex $(R, \rho)$ relative to $\mathbb{K}$, such that the canonical homomorphism $A \rightarrow \operatorname{Hom}_{\mathrm{D}(\operatorname{Mod} A)}(R, R)$ is bijective.

Proof. First we note that $\mathrm{Sq}_{\mathbb{K} / \mathbb{K}} \mathbb{K}=\mathbb{K}$, and this gives the tautological rigid com$\operatorname{plex}\left(\mathbb{K}, \rho^{\text {tau }}\right) \in \mathrm{D}_{\mathrm{f}}^{\mathrm{b}}(\operatorname{Mod} \mathbb{K})_{\mathrm{rig} / \mathbb{K}}$.

Now the structural homomorphism $\mathbb{K} \rightarrow A$ can be factored into

$$
\mathbb{K} \stackrel{f^{*}}{\longrightarrow} B \stackrel{g^{*}}{\longrightarrow} C \stackrel{h^{*}}{\longrightarrow} A,
$$

where $B:=\mathbb{K}\left[t_{1}, \ldots, t_{n}\right]$ is a polynomial algebra, $B \rightarrow C$ is surjective, and $C \rightarrow A$ is a localization. By definition $f^{\sharp} \mathbb{K}=\Omega_{B / \mathbb{K}}^{n}[n] \in \mathrm{D}_{\mathrm{f}}^{\mathrm{b}}(\operatorname{Mod} B)$, and according to Theorem 6.3 it has an induced rigidifying isomorphism $f^{\sharp}\left(\rho^{\text {tau }}\right)$. Since $B$ is a regular ring of finite Krull dimension it follows that

$$
g^{\mathrm{b}} f^{\sharp} \mathbb{K}=\operatorname{RHom}_{B}\left(C, \Omega_{B / \mathbb{K}}^{n}[n]\right) \in \mathrm{D}_{\mathrm{f}}^{\mathrm{b}}(\operatorname{Mod} C) .
$$

Since $\mathbb{K}$ is regular it follows that $g^{b} f^{\sharp} \mathbb{K}$ has finite flat dimension over $\mathbb{K}$. By Theorem 5.3 we deduce that $g^{b} f^{\sharp} \mathbb{K}$ has an induced rigidifying isomorphism $g^{b} f^{\sharp}\left(\rho^{\text {tau }}\right)$. Using Theorem 6.3 again we obtain a rigidifying isomorphism $\rho:=h^{\sharp} g^{\mathrm{b}} f^{\sharp}\left(\rho^{\mathrm{tau}}\right)$ for the complex $R:=h^{\sharp} g^{\mathrm{b}} f^{\sharp} \mathbb{K} \in \mathrm{D}_{\mathrm{f}}^{\mathrm{b}}(\operatorname{Mod} A)$.

It remains to calculate $\mathrm{RHom}_{A}(R, R)$. Since $\Omega_{B / \mathbb{K}}^{n}$ is a projective $B$-module of rank 1 we see that

$$
\operatorname{RHom}_{B}\left(f^{\sharp} \mathbb{K}, f^{\sharp} \mathbb{K}\right) \cong B .
$$

Next, by twisting, adjunction and the fact that $B$ is regular we get

$$
\begin{aligned}
& \operatorname{RHom}_{C}\left(g^{b} f^{\sharp} \mathbb{K}, g^{b} f^{\sharp} \mathbb{K}\right) \cong \operatorname{RHom}_{C}\left(g^{b} B, g^{b} B\right) \\
& \quad=\operatorname{RHom}_{C}\left(\operatorname{RHom}_{B}(C, B), \mathrm{RHom}_{B}(C, B)\right) \\
& \cong \operatorname{RHom}_{B}\left(\operatorname{RHom}_{B}(C, B), B\right) \cong C .
\end{aligned}
$$


Finally, using Proposition 1.12, and adjunction, we get

$$
\begin{aligned}
& \operatorname{RHom}_{A}\left(h^{\sharp} g^{\mathrm{b}} f^{\sharp} \mathbb{K}, h^{\sharp} g^{\mathrm{b}} f^{\sharp} \mathbb{K}\right) \cong \operatorname{RHom}_{C}\left(g^{b} f^{\sharp} \mathbb{K}, h^{\sharp} g^{b} f^{\sharp} \mathbb{K}\right) \\
& \cong A \otimes_{C} \operatorname{RHom}_{C}\left(g^{b} f^{\sharp} \mathbb{K}, g^{b} f^{\sharp} \mathbb{K}\right) \cong A \otimes_{C} C \cong A .
\end{aligned}
$$

\section{ACKNOWLEDGMENTS}

The authors wish to thank Bernhard Keller for his generous help with differential graded algebras. We also wish to thank Luchezar Avramov and Michel Van den Bergh for useful discussions and valuable suggestions. We are grateful to the referee for reading the paper carefully and making several corrections.

\section{REFERENCES}

[AFH] L. Avramov, H.-B. Foxby and S. Halperin, Differential graded homological algebra, in preparation.

[Be] K. Behrend, Differential Graded Schemes I: Perfect Resolving Algebras, eprint math.AG/0212225 at http://arxiv.org.

[DGI] W. Dwyer, J. P. C. Greenlees and S. Iyengar, Duality in algebra and topology, Adv . Math. 200 (2006), 357-402. MR2200850 (2006k:55017)

[Dr] V. Drinfeld, DG quotients of DG categories, J. Algebra 272, Number 2 (2004), 643-691. MR2028075 (2006e:18018)

[EGA] A. Grothendieck and J. Dieudonné, "Éléments de Géometrie Algébrique." Chapitre 0 IV, Publ. Math. IHES 20 (1964); Chapitre IV, Publ. Math. IHES 32 (1967). MR0173675 (30:3885)

[FIJ] A. Frankild, S. Iyengar and P. Jørgensen, Dualizing Differential Graded Modules and Gorenstein Differential Graded Algebras, J. London Math. Soc. (2) 68 (2003), 288-306. MR1994683 (2004f:16013)

[Hi] V. Hinich, Homological algebra of homotopy algebras, Comm. Algebra 25 (1997), no. 10, 3291-3323. MR1465117 (99b:18017)

[Ke] B. Keller, Deriving DG categories, Ann. Sci. École Norm. Sup. (4) 27 (1994), no. 1, 63-102. MR1258406 (95e:18010)

[KM] I. Kriz and J.P. May, "Operads, Algebras, Modules and Motives," Astérisque 233 (1995). MR1361938 (96j:18006)

[KS] M. Kashiwara and P. Schapira, "Sheaves on Manifolds," Springer-Verlag, Berlin, 1990. MR1074006 (92a:58132)

[ML] S. Maclane, "Homology," Springer-Verlag, 1994 (reprint). MR1344215 (96d:18001)

[Ne] A. Neeman, The Grothendieck duality theorem via Bousfield's techniques and Brown representability, J. Amer. Math. Soc. 9 (1996), 205-236. MR1308405 (96c:18006)

[Qu] D. Quillen, On the cohomology of commutative rings, Proc. Symp. Pure Math. (AMS) 17 (1970), pp. 65-87. MR0257068 (41:1722)

[RD] R. Hartshorne, "Residues and Duality," Lecture Notes in Math. 20, Springer-Verlag, Berlin, 1966. MR0222093 (36:5145)

[Sp] N. Spaltenstein, Resolutions of unbounded complexes, Compositio Math. 65 (1988), no. 2, 121-154. MR932640 (89m:18013)

[Ta] G. Tabuada, Une structure de catégorie de modèles de Quillen sur la catégorie des dgcatégories, C. R. Acad. Sci. Paris, Ser. I 340 (2005), 15-19. MR2112034 (2005h:18033)

[VdB] M. Van den Bergh, Existence theorems for dualizing complexes over non-commutative graded and filtered rings, J. Algebra 195 (1997), no. 2, 662-679. MR1469646 (99b:16010)

[Ye1] A. Yekutieli, Dualizing Complexes over Noncommutative Graded Algebras, J. Algebra 153 no. 1 (1992), 41-84. MR1195406 (94a:16077)

[Ye2] A. Yekutieli, "An Explicit Construction of the Grothendieck Residue Complex" (with an appendix by P. Sastry), Astérisque 208 (1992). MR1213064 (94e:14026)

[Ye3] A. Yekutieli, Smooth formal embeddings and the residue complex, Canadian J. Math. 50 (1998), 863-896. MR1638635 (99i:14004) 
[Ye4] A. Yekutieli, Rigid Dualizing Complexes and Perverse Coherent Sheaves on Schemes, in preparation.

[YZ1] A. Yekutieli and J.J. Zhang, Rings with Auslander dualizing complexes, J. Algebra 213 (1999), no. 1, 1-51. MR1674648 (2000f:16012)

[YZ2] A. Yekutieli and J.J. Zhang, Residue complexes over noncommutative rings, J. Algebra 259 (2003) no. 2, 451-493. MR1955528 (2004a:16010)

[YZ3] A. Yekutieli and J.J. Zhang, Dualizing complexes and perverse modules over differential algebras, Compositio Math. 141 (2005), 620-654. MR2135281 (2006c:16014)

[YZ4] A. Yekutieli and J.J. Zhang, Rigid Dualizing Complexes over Commutative Rings, to appear in Algebr. Represent. Theory, eprint math.AG/0601654 at http://arxiv.org.

Department of Mathematics, Ben Gurion University, Be'er Sheva 84105, Israel

E-mail address: amyekut@math.bgu.ac.il

Department of Mathematics, Box 354350, University of Washington, Seattle, WashINGTON 98195

E-mail address: zhang@math.washington.edu 